Homology, Homotopy and Applications, vol.22(2), 2020, pp.221-250

\title{
COMPATIBLE ACTIONS IN SEMI-ABELIAN CATEGORIES
}

\section{DAVIDE DI MICCO AND TIM VAN DER LINDEN}

\author{
(communicated by Ronald Brown)
}

\begin{abstract}
The concept of a pair of compatible actions was introduced in the case of groups by Brown and Loday [6] and in the case of Lie algebras by Ellis [14]. In this article we extend it to the context of semi-abelian categories (that satisfy the Smith is Huq condition). We give a new construction of the Peiffer product, which specialises to the definitions known for groups and Lie algebras. We use it to prove our main result, on the connection between pairs of compatible actions and pairs of crossed modules over a common base object. We also study the Peiffer product in its own right, in terms of its universal properties, and prove its equivalence with existing definitions in specific cases.
\end{abstract}

\section{Introduction}

The concept of a pair of compatible actions was first introduced in the category of groups by Brown and Loday, in relation to their work on the non-abelian tensor product of groups [6]. Later, in [14], the definition was adapted to the context of Lie algebras, where it was further studied in $[\mathbf{2 6}, \mathbf{1 3}]$. Since then, several other particular instances of compatible actions have been defined or used, in various settings: see for example $[\mathbf{1 7}, \mathbf{9}, \mathbf{8}]$, and $[\mathbf{2 2}, \mathbf{2 3}]$ where the non-abelian derived functors of $\otimes$ were studied.

The aim of this article is to provide a general definition in semi-abelian categories (in the sense of [25]), in a way that extends these as special cases. In particular, this will give us the tools to develop a unified theory, in such a way that computing the non-abelian tensor product of compatible actions is the same as computing the non-abelian tensor product of internal crossed modules. This process generalises the diverse particular notions of non-abelian tensor product that appear in the literature so far.

With this idea in mind, we first examine the case of groups from a new perspective, aiming to use a diagrammatic and internal approach whenever this is possible. To do so, we take advantage of the equivalence between group actions in the usual sense and internal actions (introduced in $[5,2]$ ) in the category $\mathbf{G r p}$, as well as the equivalence

The second author is a Research Associate of the Fonds de la Recherche Scientifique-FNRS.

Received August 13, 2019, revised December 5, 2019; published on April 29, 2020.

2010 Mathematics Subject Classification: 18D35, 18E10, 20 J15.

Key words and phrases: semi-abelian category, pair of compatible actions, internal action, crossed module, Peiffer product, non-abelian tensor product.

Article available at http://dx.doi.org/10.4310/HHA.2020.v22.n2.a14

Copyright (c) 2020, International Press. Permission to copy for private use granted. 
between crossed modules of groups and internal crossed modules in Grp (see [24]). Thus we may separate properties which are specific for groups from those that are purely categorical.

The conditions which we single out in the category Grp in terms of the internal action formalism become our general definition of "a pair of compatible actions". This definition makes sense as soon as the surrounding category is semi-abelian. However, we shall always assume that the so-called Smith is Huq condition ( $\mathrm{SH}$ ) holds as well. This is a relatively mild condition which excludes loops, for instance, but includes all categories of groups with operations; see [28, 10]. This simplifies our work, since under (SH) internal crossed modules allow an easier description [24, 28].

Our main tool is an extension, to the semi-abelian context, of the Peiffer product $M \bowtie N$ of two objects $M$ and $N$ acting on each other (via an action $\xi_{M}^{N}$ of $N$ on $M$ and an action $\xi_{N}^{M}$ of $M$ on $N$ ). A notion of Peiffer product has already been introduced in [11], in the special case of a pair of internal precrossed modules over a common base object. Ours, however, is a different approach, and a priori the two notions do not coincide. Our definition is a direct generalisation of the group and Lie algebra versions of the Peiffer product, which were originally introduced respectively in [32] and in [14]. It is well defined as soon as the two objects $M$ and $N$ act on each other, whereas for the definition in [11] they also need to satisfy some compatibility conditions. Moreover, when the actions $\xi_{M}^{N}$ and $\xi_{N}^{M}$ are compatible, the Peiffer product $M \bowtie N$ is endowed with internal crossed module structures $\left(M \stackrel{l_{M}}{\longrightarrow} M \bowtie N, \xi_{M}^{M \bowtie N}\right)$ and $\left(N \stackrel{l_{N}}{\longrightarrow} M \bowtie N, \xi_{N}^{M \bowtie N}\right)$.

We use this as an ingredient in the generalisation of a result, stated in [6] for groups and in [26] for Lie algebras, to any semi-abelian category that satisfies the condition (SH). We show namely that two objects $M$ and $N$ act on each other compatibly if and only if there exists a third object $L$ endowed with two internal crossed module structures $\left(M \stackrel{\mu}{\rightarrow} L, \xi_{M}^{L}\right)$ and $\left(N \stackrel{\nu}{\rightarrow} L, \xi_{N}^{L}\right)$. Amongst other things, this allows us to deduce that our definition of compatibility for pairs of internal actions restricts to the classical definitions for groups and Lie algebras. Another consequence of this result is that the non-abelian tensor product introduced in the forthcoming article [12] has two natural interpretations: either as a tensor product of compatible internal actions, or equivalently as a tensor product of crossed modules over a common base object.

Finally, we study the Peiffer product via its universal properties. We also prove that, under the additional hypothesis of algebraic coherence [10], our definition of the Peiffer product coincides with the one given in [11].

\section{Structure of the text}

The paper is organised as follows. In the first section we collect preliminary definitions and results on internal actions in semi-abelian categories. We recall the definitions of the bifunctors $b$ and $\diamond$ as well as some related constructions. For a given semi-abelian category $\mathbb{A}$, we describe the category of points in $\mathbb{A}$ and the category of internal actions in $\mathbb{A}$, together with the equivalence between the two.

In Section 3 we examine the concept of a pair of compatible actions in the category of groups. First we consider the definition of compatibility given in [6] and we translate it into its diagrammatic form. Then we construct the Peiffer product as a coequaliser and we prove that it coincides with the definition already known for the case of 
groups. In Proposition 3.10 we prove a result stated in [6], namely that two groups $M$ and $N$ act on each other compatibly if and only if there exists a third group $L$ endowed with two crossed module structures $\left(M \stackrel{\mu}{\rightarrow} L, \xi_{M}^{L}\right)$ and $\left(N \stackrel{\nu}{\rightarrow} L, \xi_{N}^{L}\right)$.

Section 4 contains our main results. We work in the context of a semi-abelian category $\mathbb{A}$ that satisfies the Smith is Huq condition ( $\mathrm{SH}$ ). We express the definition of compatibility in this general context and show in Proposition 4.3 that whenever we have a pair of internal crossed modules over a common base object, they induce a pair of compatible internal actions.

Then we construct the Peiffer product of two internal actions in three distinct ways: first we imitate what happens in the case of groups, constructing the Peiffer product for each pair of objects acting on each other. In Proposition 4.5 we prove that this is the same as taking the pushout of the two semi-direct products. Then we give a more specific definition that requires the actions to be compatible. Finally, we show in Proposition 4.8 that, if the compatibility conditions are satisfied, then the two definitions coincide.

We prove in Proposition 4.9 that whenever the actions are compatible, their Peiffer product is automatically endowed with internal crossed module structures $\left(M \stackrel{l_{M}}{\longrightarrow} M \bowtie N, \xi_{M}^{M \bowtie N}\right)$ and $\left(N \stackrel{l_{N}}{\longrightarrow} M \bowtie N, \xi_{N}^{M \bowtie N}\right)$. This leads to Theorem 4.11, which is a generalisation to semi-abelian categories of Proposition 3.10, proven for groups in the previous section: two objects $M$ and $N$ act on each other compatibly if and only if there exists a third object $L$ endowed with two internal crossed module structures $\left(M \stackrel{\mu}{\rightarrow} L, \xi_{M}^{L}\right)$ and $\left(N \stackrel{\nu}{\rightarrow} L, \xi_{N}^{L}\right)$. Via this result we obtain Corollary 4.12 and Corollary 4.13 as confirmations of the equivalence between our general definition of compatibility and the specific ones in the cases of groups and Lie algebras.

We conclude the paper with a study of the Peiffer product via its universal properties (Section 5, in particular, Proposition 5.1 and Proposition 4.5). Here we also prove that, under the additional hypothesis of algebraic coherence [10], our definition of the Peiffer product coincides with the one given in [11]. Via results in [11], this further implies that under an additional condition called (UA), the actions induced by two $L$-crossed module structures have a Peiffer product which is again an $L$-crossed module; furthermore, it is the coproduct in $\operatorname{XMod}_{L}(\mathbb{A})$ of the given $L$-crossed modules. This generalises Proposition 3.4 in [13].

\section{Preliminaries on internal actions}

In what follows, we let $\mathbb{A}$ be a semi-abelian category [25]: pointed, Barr exact, Bourn protomodular with binary coproducts. This concept unifies earlier attempts (including, for instance, $[21,15,30]$ ) at providing a categorical framework that extends the context of abelian categories to include non-additive categories of algebraic structures such as groups, Lie algebras, loops, rings, etc. In this setting, the basic lemmas of homological algebra - the $3 \times 3$ Lemma, the Short Five Lemma, the Snake Lemma - hold [3, 1].

The category of internal actions $\operatorname{Act}(\mathbb{A})$ and the category of internal crossed modules $\mathbf{X M o d}(\mathbb{A})$ in any semi-abelian category $\mathbb{A}$ are again semi-abelian. We give a quick overview of some important definitions.

Recall that a regular epimorphism is a coequalizer of some pair of arrows. Any 
semi-abelian category is a regular category, which means that finite limits and coequalisers of kernel pairs exist, and regular epimorphisms are pullback-stable. A Barr exact category is a regular category in which every equivalence relation is a kernel pair. In a pointed setting, Bourn protomodularity is the condition that the Split Short Five Lemma holds.

Definition 2.1 ([4]). A regular pushout is a commutative square of regular epimorphisms as on the left
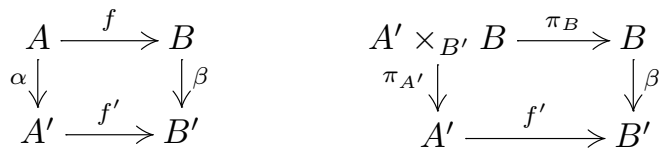

such that the comparison morphism $\langle\alpha, f\rangle: A \rightarrow A^{\prime} \times{ }_{B^{\prime}} B$ to the induced pullback square on the right is a regular epimorphism as well.

It is well known that in a semi-abelian category, a commutative square of regular epimorphisms is a regular pushout if and only if it is a pushout. In fact, this characterises semi-abelian categories amongst finitely cocomplete homological categories (in the sense of [1]: pointed, regular, protomodular). Regular pushouts can be recognised as follows:

Lemma $2.2([4])$. Consider a square of regular epimorphisms in a homological category and take kernels to the left as in the diagram

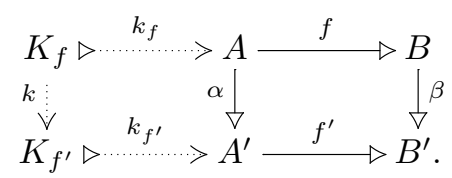

The induced morphism $k$ is a regular epimorphism if and only if the given square is a regular pushout.

\subsection{The bifunctor $b$}

For an object $A$ in a semi-abelian category $\mathbb{A}$, internal $A$-actions are defined as algebras over a certain monad $A b(-)$.

Definition 2.3. The bifunctor $b: \mathbb{A} \times \mathbb{A} \rightarrow \mathbb{A}$ is defined on objects as the kernel

$$
A b B \stackrel{k_{A, B}}{\longrightarrow} A+B \stackrel{\left(\begin{array}{c}
1_{A} \\
0
\end{array}\right)}{\longrightarrow} A .
$$

Using the universal property of kernels, its behaviour on arrows is determined by

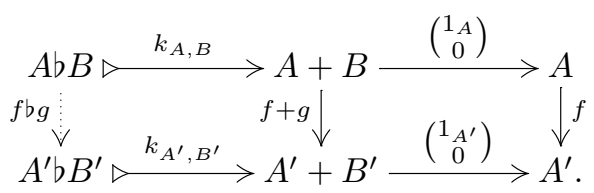

Example 2.4. In the category $\operatorname{Grp}$ the coproduct $A+B$ is the so-called free product of $A$ and $B$, the group freely generated by the disjoint union of $A$ and $B$, modulo 
the relations that hold in $A$ or in $B$. This means that an element in $A+B$ can be represented as a word obtained by juxtaposition of elements in $A$ and in $B$. Then it is easy to deduce that $A b B$ is the subgroup of $A+B$ whose elements are represented by the words of the form $a_{1} b_{1} \cdots a_{n} b_{n}$ such that $a_{1} \cdots a_{n}=1 \in A$. Furthermore, it can be shown that each word in $A b B$ can be written as a juxtaposition of formal conjugations, that is

$$
A b B=\left\langle a b a^{-1} \mid a \in A, b \in B\right\rangle .
$$

The following example expresses the idea of the proof, which easy generalises to any word in $A b B$.

$$
\begin{aligned}
a_{1} b_{1} a_{2} b_{2} a_{3} b_{3} & =\left(a_{1} b_{1} a_{1}^{-1}\right)\left(a_{1} a_{2} b_{2} a_{2}^{-1} a_{1}^{-1}\right)\left(a_{1} a_{2} a_{3}\right) b_{3} \\
& =\left(a_{1} b_{1} a_{1}^{-1}\right)\left(a_{1} a_{2} b_{2}\left(a_{1} a_{2}\right)^{-1}\right) 1\left(1 b_{3} 1^{-1}\right) .
\end{aligned}
$$

Remark 2.5. For any fixed object $A \in \mathbb{A}$, the triple $\left(A b(-), \eta^{A}, \mu^{A}\right)$ is a monad, where for $A, B \in \mathbb{A}$ we define $\eta_{B}^{A}: B \rightarrow A b B$ as in

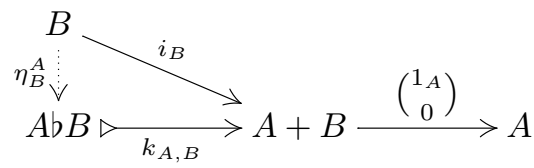

and $\mu_{B}^{A}: A b(A b B) \rightarrow A b B$ as in

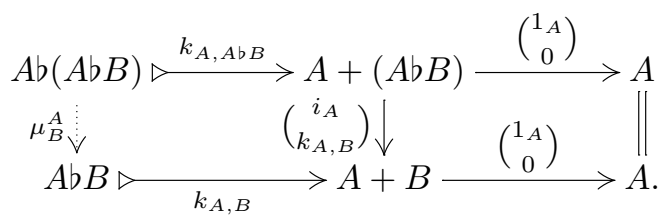

Lemma 2.6. In a semi-abelian category, consider regular epimorphisms $\alpha: A \rightarrow A^{\prime}$ and $\beta: B \rightarrow B^{\prime}$. Then both $\alpha+\beta$ and $\alpha b \beta$ are regular epimorphisms as well.

Proof. The first statement is easily shown checking that, if $\alpha=\operatorname{coeq}\left(x_{1}, x_{2}\right)$ and $\beta=\operatorname{coeq}\left(y_{1}, y_{2}\right)$, then $\alpha+\beta=\operatorname{coe} q\left(x_{1}+y_{1}, x_{2}+y_{2}\right)$. For what regards the second statement we build the diagram

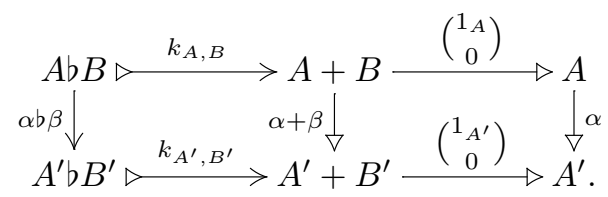

Thanks to Lemma 2.2 it suffices to show that the right-hand square is a pushout in order to obtain that $\alpha b \beta$ is a regular epimorphism as well. This is easy to do by direct verification of the universal property of pushouts.

\subsection{The cosmash product $\diamond$}

Cosmash products [7] may be used to define commutators [27, 19] and may help expressing properties of internal actions. We start by exploring the relationship with $b$. 
Definition 2.7. Given two objects $A$ and $B$ in $A$, consider the morphism

$$
\Sigma_{A, B}=\left(\begin{array}{c}
\left\langle 1_{A}, 0\right\rangle \\
\left\langle 0,1_{B}\right\rangle
\end{array}\right)=\left\langle\left(\begin{array}{c}
1_{A} \\
0
\end{array}\right),\left(\begin{array}{c}
0 \\
1_{B}
\end{array}\right)\right\rangle: A+B \longrightarrow A \times B .
$$

Since $\mathbb{A}$ is semi-abelian, the morphism $\Sigma_{A, B}$ is a regular epimorphism. (This is actually true in the much more general context of a unital category: see [1].) By taking its kernel we find the short exact sequence

$$
0 \longrightarrow A \diamond B \triangleright \stackrel{h_{A, B}}{\longrightarrow} A+B \stackrel{\Sigma_{A, B}}{\longrightarrow} A \times B \longrightarrow 0
$$

where $A \diamond B$ is called the cosmash product of $A$ and $B$.

Remark 2.8. Notice that the inclusion of $A \diamond B$ into $A+B$ factors through $A b B$, because the latter is the kernel of $\left(\begin{array}{c}1_{A} \\ 0\end{array}\right): A+B \rightarrow A$. Moreover, we have another split short exact sequence involving the cosmash product, namely

$$
0 \longrightarrow A \diamond B \stackrel{i_{A, B}}{\longrightarrow} A b B \underset{\eta_{B}^{A}}{\stackrel{\tau_{B}^{A}}{\rightleftarrows}} B \longrightarrow 0
$$

where $\tau_{B}^{A}:-\left(\begin{array}{c}0 \\ 1_{B}\end{array}\right) \circ k_{A, B}$ is the so-called trivial action of $A$ on $B$ : see Example 2.18 where this point of view is explained in detail. The exactness of the sequence can be shown by constructing the $3 \times 3$ diagram

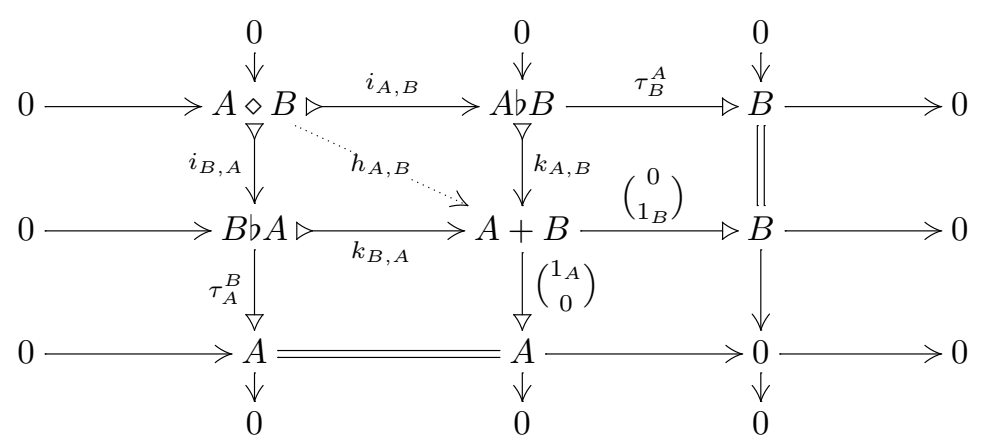

from the bottom-right square by taking kernels, and then by noticing that the top-left object is the kernel of the comparison morphism from $A+B$ to the pullback induced by the lower-right square: since this morphism is precisely $\Sigma_{A, B}$, its kernel is $A \diamond B$.

Moreover, the upper left square is a pullback and hence $A \diamond B$ can be seen as the intersection of the subobjects $A b B$ and $B b A$ of $A+B$. Furthermore, since $\mathbb{A}$ is semiabelian, in the split short exact sequence (1) the morphisms $i_{A, B}$ and $\eta_{B}^{A}$ are jointly extremal-epimorphic [1, Lemma 3.1.22]. Thus we obtain the regular epimorphism

$$
\left(\begin{array}{c}
i_{A, B} \\
\eta_{B}^{A}
\end{array}\right):(A \diamond B)+B \rightarrow A b B .
$$

Lemma 2.9. Let $X$ be an object in a semi-abelian category $\mathbb{A}$. Then the functor $(-) b X: \mathbb{A} \rightarrow \mathbb{A}$ preserves coequalisers of reflexive graphs.

Proof. Consider a reflexive graph with its coequaliser

$$
A \underset{c}{\stackrel{d}{\rightleftarrows}} B \stackrel{q}{\longrightarrow} \longrightarrow Q
$$


and the induced diagram

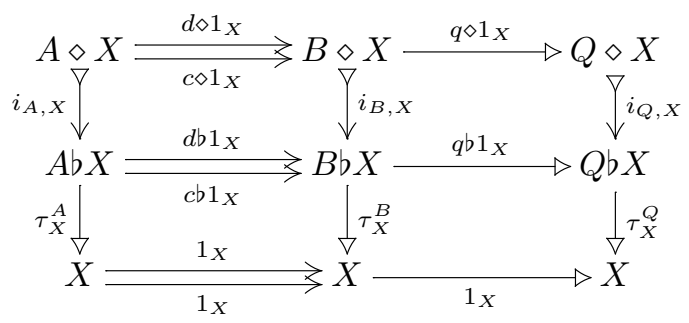

By using Corollary 2.27 in the first arXiv version of [19] we know that $q \diamond 1_{X}$ is again the coequaliser of $d \diamond 1_{X}$ and $c \diamond 1_{X}$. We already know that $q b 1_{X}$ is a regular epimorphism by Lemma 2.6 and that $\left(q b 1_{X}\right) \circ\left(d b 1_{X}\right)=\left(q b 1_{X}\right) \circ\left(c b 1_{X}\right)$, so it remains to show the universal property.

First of all, by examining the squares on the right, we can see that they form a horizontal morphism of vertical short exact sequences, and since $1_{X}$ is an isomorphism, we conclude that the top square is a pullback. This implies that it is also a pushout: indeed when we take kernels horizontally we obtain an induced isomorphism between them, which in turn implies that the given square is a pushout.

Now suppose that there exists a morphism $z: B b X \rightarrow Z$ such that $z \circ\left(d b 1_{X}\right)=$ $z \circ\left(c b 1_{X}\right)$. Then $z \circ i_{B, X} \circ\left(d \diamond 1_{X}\right)=z \circ i_{B, X} \circ\left(c \diamond 1_{X}\right)$ and hence there is a unique morphism $\phi: Q \diamond X \rightarrow Z$ such that $\phi \circ\left(q \diamond 1_{X}\right)=z \circ i_{B, X}$. Our claim now follows from the universal property of the pushout.

\subsection{The ternary cosmash product}

Following [20], in [19] Hartl and Van der Linden define the $n$-ary version of the cosmash product. We are interested in the ternary case, and in some relations between it and the binary case.

Definition 2.10. Given three objects $A, B$ and $C$ in $A$, consider the morphism

$$
\Sigma_{A, B, C}=\left(\begin{array}{ccc}
i_{A} & i_{A} & 0 \\
i_{B} & 0 & i_{B} \\
0 & i_{C} & i_{C}
\end{array}\right): A+B+C \longrightarrow(A+B) \times(A+C) \times(B+C) .
$$

Its kernel is written

$$
A \diamond B \diamond C \triangleright \stackrel{h_{A, B, C}}{\longrightarrow} A+B+C
$$

and it is called the ternary cosmash product of $A, B$ and $C$. Like in the binary case, it is obvious that, up to isomorphism, the ternary cosmash product does not depend on the order of the objects.

In [19] the authors define folding operations linking cosmash products of different arities: for our purposes we only need to recall one of them.

Definition 2.11. Given two objects $A$ and $B$ we can construct a morphism

$$
S_{2,1}^{A, B}: A \diamond A \diamond B \rightarrow A \diamond B
$$


through the diagram

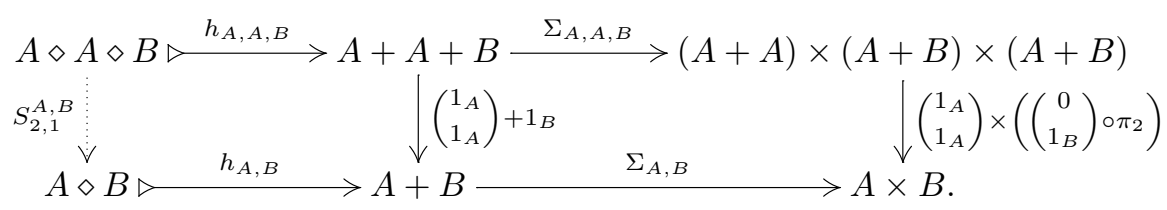

The symmetry in the definition of cosmash products allows for numerous straightforward variations on this theme; in particular, we write $S_{2,1}^{A, B}: A \diamond B \diamond A \rightarrow A \diamond B$ (two $A$ s and one $B$, replace the middle morphism with the one induced by $\iota_{A}, \iota_{B}$ and $\iota_{A}$, and the one on the right by the projection on the last two factors followed by $\left.\left(\begin{array}{c}1_{A} \\ 1_{A}\end{array}\right) \times\left(\begin{array}{c}0 \\ 1_{B}\end{array}\right)\right)$ and $S_{1,2}^{A, B}: A \diamond B \diamond B \rightarrow A \diamond B$ (two $B$ s and one $A$, so use $\left(\begin{array}{c}1_{B} \\ 1_{B}\end{array}\right)$ instead of $\left.\left(\begin{array}{l}1_{A} \\ 1_{A}\end{array}\right)\right)$. We also need the following morphism from $A \diamond B \diamond C$ to $(A+B) b C$.

Definition 2.12. Consider the object $(A+B) b C$ and define the morphism $j_{A, B, C}$ as in the diagram

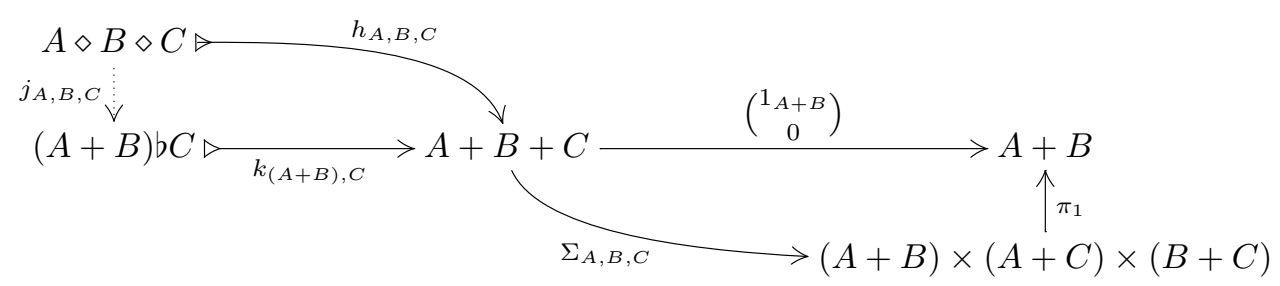

In particular, if $A=B$, then we have the commutative diagram

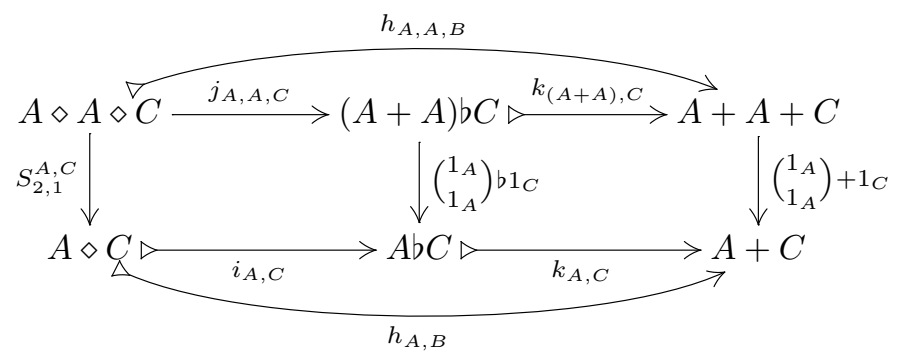

Lemma 2.13. It is possible to cover the object $(A+B) b C$ with the three components $(A \diamond B \diamond C),(A b C)$ and $(B b C)$.

Proof. By Lemma 2.12 in [19] we know that there is a regular epimorphism of the form

$$
(A \diamond B \diamond C)+(A \diamond C)+(B \diamond C) \longrightarrow \quad e \quad \triangleright(A+B) \diamond C .
$$

Using Remark 2.8 we are able to construct the square

$$
\begin{aligned}
(A \diamond B \diamond C)+(A \diamond C)+(B \diamond C)+C+C \stackrel{1+\left(\begin{array}{c}
i_{A, C} \\
\eta_{C}^{A}
\end{array}\right)+\left(\begin{array}{c}
i_{B, C} \\
\eta_{C}^{B}
\end{array}\right)}{\longrightarrow}(A \diamond B \diamond C)+(A b C)+(B b C) \\
\begin{array}{c}
e+\left.\left(\begin{array}{c}
1_{C} \\
1_{C}
\end{array}\right)\right|_{\nabla} \\
((A+B) \diamond C)+C \longrightarrow\left(\begin{array}{c}
j_{A, B, C} \\
i_{A} b 1_{C} \\
i_{B} b 1_{C}
\end{array}\right)
\end{array} \\
\left(\begin{array}{c}
i_{(A+B), C} \\
\eta_{C}^{A+B}
\end{array}\right)
\end{aligned}
$$


from which we see that the vertical morphism on the right is a regular epimorphism.

\subsection{The categories $\operatorname{Pt}(\mathbb{A})$ and $\operatorname{Act}(\mathbb{A})$}

In semi-abelian categories there is a concept of internal action, which via a semidirect product construction is equivalent to the concept of a point - a split epimorphism with a chosen splitting.

Definition 2.14. A point $(p, s)$ in $\mathbb{A}$ is a split epimorphism $p$ with a chosen splitting $s$, that is $p: A \rightarrow B$ and $s: B \rightarrow A$ such that $p \circ s=1_{B}$. A morphism of points $(p, s) \rightarrow$ $\left(p^{\prime}, s^{\prime}\right)$ is given by a pair of vertical morphisms $(f, g)$ such that the two squares formed by parallel morphisms

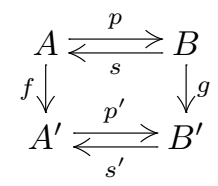

commute. $\operatorname{Pt}(\mathbb{A})$ is the category of points in $\mathbb{A}$ and morphisms between them. Since the codomain of $p$ is $B$, the point $(p, s)$ is also called a point over $B$.

Having described the category of points, we now shift to internal actions, whose category is equivalent to the former whenever the base category $\mathbb{A}$ is semi-abelian [5].

Definition 2.15. An internal action of $A$ on $X$ (or simply $A$-action or action) in $A$ is a triple $(A, X, \xi)$ with $\xi: A b X \rightarrow X$ a morphism in $\mathbb{A}$ such that $(X, \xi)$ is an algebra for the monad $A b(-): \mathbb{A} \rightarrow \mathbb{A}$. This means that the diagrams

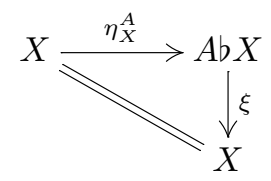

and

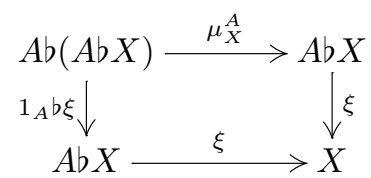

- notations as in Remark 2.5-commute. A morphism of actions from $(A, X, \xi)$ to $\left(A^{\prime}, X^{\prime}, \xi^{\prime}\right)$ is given by a pair $(f, g)$ of morphisms in $\mathbb{A}$, with $f: A \rightarrow A^{\prime}$ and $g: X \rightarrow$ $X^{\prime}$, such that $\xi^{\prime} \circ(f b g)=g \circ \xi$. The category of actions and morphisms between them is denoted by $\operatorname{Act}(\mathbb{A})$.

Example 2.16. If we fix $\mathbb{A}=\mathbf{G r p}$ we find that internal actions coincide with the usual group actions. Indeed due to Example 2.4, in order to define such an internal action $\xi: A b X \rightarrow X$ it suffices to specify where the elements of the form $a x a^{-1}$ are sent, since they generate the whole subgroup $A b X$. Now an internal action $\xi$ corresponds to the group action $\psi: A \times X \rightarrow X$ given by $\psi(a, x):-\xi\left(a x a^{-1}\right)$. Conversely, starting from a group action $\psi$ we define $\xi: A b X \rightarrow X$ on the generators by $\xi\left(a x a^{-1}\right):-\psi(a, x)$. It is easy to show that these are actions in the appropriate sense. ( $\xi$ being a morphism and the axioms for it to be an internal action amount to the group action axioms for the function $\psi$.) The correspondence just depicted determines an equivalence between internal actions in Grp and group actions.

Remark 2.1\%. Whenever the base category $\mathbb{A}$ is semi-abelian we have an equivalence $\operatorname{Pt}(\mathbb{A}) \simeq \operatorname{Act}(\mathbb{A})$. The functor $\mathbf{P t}(\mathbb{A}) \rightarrow \operatorname{Act}(\mathbb{A})$ sends a point $(p: A \rightarrow B, s: B \rightarrow A)$ 
to the action $\left(B, K_{p}, \xi\right)$, where $\xi$ is the unique morphism making the diagram

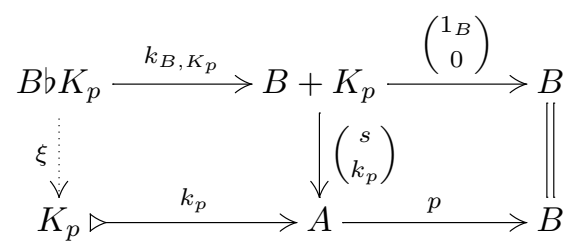

commute. The functor $\operatorname{Act}(\mathbb{A}) \rightarrow \mathbf{P t}(\mathbb{A})$ sends an action $(A, X, \xi)$ to the point

$$
X \rtimes_{\xi} A \underset{i_{\xi}}{\stackrel{\pi_{\xi}}{\rightleftarrows}} A,
$$

where the semi-direct product $X \rtimes_{\xi} A$ is defined as the coequaliser

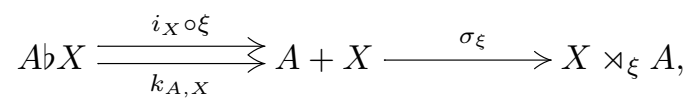

the morphism $\pi_{\xi}$ is the unique morphism such that

$$
A+X \underset{\pi_{A, X}}{\stackrel{\sigma_{\xi}}{\longrightarrow}} X \rtimes_{A}^{\rtimes_{\xi} A}
$$

commutes, and, finally, $i_{\xi}=\sigma_{\xi} \circ i_{A}$. We will sometimes write $X \rtimes_{\xi} A$ as $X \rtimes A$, when there is no risk of confusion regarding the action involved. Notice that the morphism

$$
k:-\sigma_{\xi} \circ i_{X}: X \rightarrow X \rtimes_{\xi} A
$$

is the kernel of $\pi_{\xi}$ : it is easy to see that $\pi_{\xi} \circ k=0$, whereas for the universal property some work needs to be done-see, for instance, [29].

Example 2.18. The trivial action $(A, X, \tau)$ is given by

$$
\tau=\left(\begin{array}{c}
0 \\
1_{X}
\end{array}\right) \circ k_{A, X}: A b X \rightarrow X .
$$

Then we have that $\left(X \rtimes_{\tau} A, \sigma_{\tau}\right) \cong \operatorname{Coeq}\left(i_{X} \circ\left(\left(\begin{array}{c}0 \\ 1_{X}\end{array}\right) \circ k_{A, X}\right), k_{A, X}\right)$. Both $\left(\begin{array}{c}1_{A} \\ 0\end{array}\right)$ and $\left(\begin{array}{c}0 \\ 1_{X}\end{array}\right)$ coequalise these two morphisms, so (following the example of the trivial action in Grp) a first guess would be that

$$
\operatorname{Coeq}\left(i_{X} \circ\left(\begin{array}{c}
0 \\
1_{X}
\end{array}\right) \circ k_{A, X}, k_{A, X}\right) \cong\left(A \times X,\left\langle\left(\begin{array}{c}
1_{A} \\
0
\end{array}\right),\left(\begin{array}{c}
0 \\
1_{X}
\end{array}\right)\right\rangle\right) \text {. }
$$

In order to prove this, we may use the equivalence $\operatorname{Pt}(\mathbb{A}) \simeq \operatorname{Act}(\mathbb{A})$. In particular, we claim that the desired point is given by $\left(\pi_{A}: A \times X \rightarrow A,\left\langle 1_{A}, 0\right\rangle: A \rightarrow A \times X\right)$ and hence it suffices to show that $\tau=\left(\begin{array}{c}0 \\ 1_{X}\end{array}\right) \circ k_{A, X}$ makes the diagram

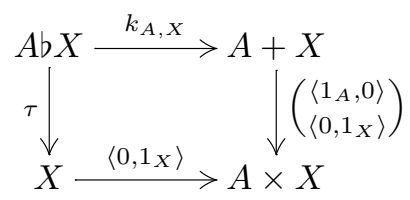

commute. This is done by direct and easy calculations. 
Example 2.19. The conjugation action $\left(A, A, \chi_{A}\right)$ is given by

$$
\chi_{A}=\left(\begin{array}{l}
1_{A} \\
1_{A}
\end{array}\right) \circ k_{A, A}: A b A \rightarrow A .
$$

Then we have that $\left(A \rtimes_{\chi_{A}} A, \sigma_{\chi_{A}}\right) \cong \operatorname{Coeq}\left(i_{2} \circ\left(\left(\begin{array}{c}1_{A} \\ 1_{A}\end{array}\right) \circ k_{A, A}\right), k_{A, A}\right)$. Both $\left(\begin{array}{c}1_{A} \\ 0\end{array}\right)$ and $\left(\begin{array}{l}1_{A} \\ 1_{A}\end{array}\right)$ coequalise these two morphisms, so a first guess (again following the example of Grp) would be that

$$
\operatorname{Coeq}\left(i_{2} \circ\left(\begin{array}{c}
1_{A} \\
1_{A}
\end{array}\right) \circ k_{A, A}, k_{A, A}\right) \cong\left(A \times A,\left\langle\left(\begin{array}{c}
1_{A} \\
0
\end{array}\right),\left(\begin{array}{c}
1_{A} \\
1_{A}
\end{array}\right)\right\rangle\right) .
$$

In order to prove this, we use the same strategy as in the previous example.

Remark 2.20. Notice that, by the definition of the semi-direct product, it is easy to show that the diagram on the left
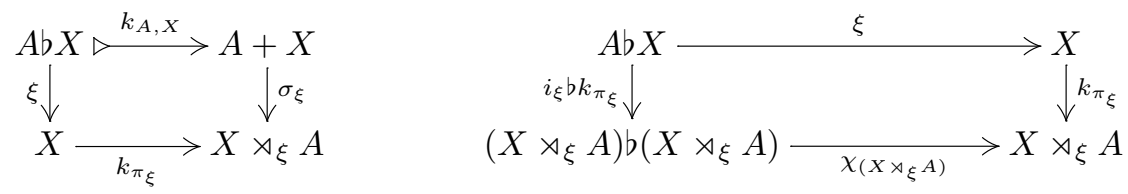

is a pushout. Thanks to this commutativity we can show that also the square on the right commutes, which means that "computing an action" is the same as "computing the conjugation in the induced semi-direct product".

Remark 2.21. Notice that, if $(B, X, \xi: B b X \rightarrow X)$ is an action and $f: A \rightarrow B$ is any morphism, then also $\left(A, X, \xi \circ\left(f b 1_{X}\right): A b X \rightarrow X\right)$ is an action. Indeed the diagrams
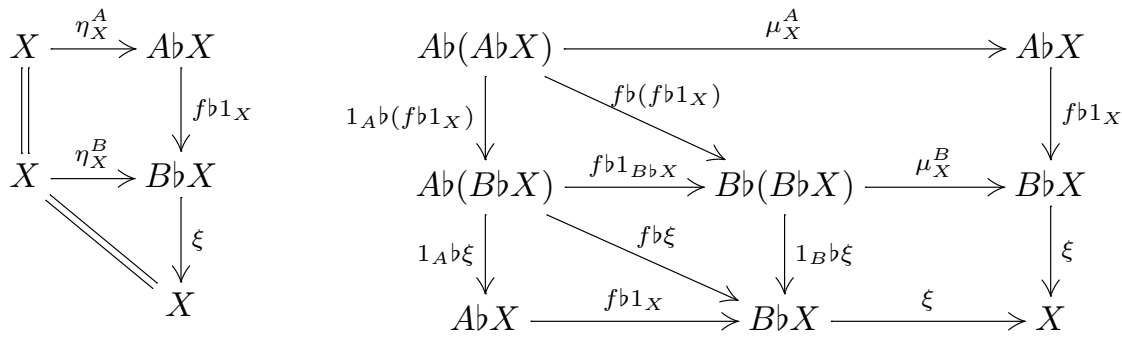

commute. The action $\xi \circ\left(f b 1_{X}\right)$ is often called pullback action of $\xi$ along $f$ and the reason is the following. Consider the diagram

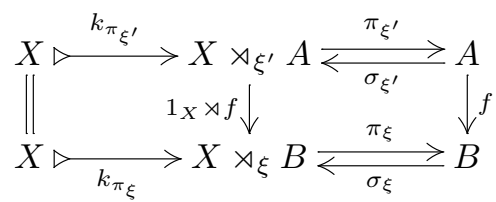

where the bottom row is the point associated to $\xi$, whereas the first row is obtained taking the pullback of $\pi_{\xi}$ along $f$. Then it is easy to see that the action $\xi^{\prime}$ coincides with $\xi \circ\left(f b 1_{X}\right)$.

Remark 2.22. In order to recover a point over $B$, in general slightly less is needed than a $B b(-)$-algebra structure. Every time we have an action $\xi: A b X \rightarrow X$ we 
can construct the corresponding action core ${ }^{\diamond} \xi: A \diamond X \rightarrow X$ as the composition of $\xi$ and $i_{A, X}: A \diamond X \rightarrow A b X$. Action cores (morphisms $A \diamond X \rightarrow X$ that satisfy suitable axioms) were defined and studied in $[\mathbf{1 9}, \mathbf{1 8}]$. The main point is that, in the semiabelian context, from an action core ${ }^{\diamond} \xi: A \diamond X \rightarrow X$ we can recover the action $\xi$. Furthermore, crossed module structures can be expressed in terms of action cores.

Example 2.23. Consider an action $\xi: A b X \rightarrow X$ in Grp, sending a generator $a x a^{-1}$ of $A b X$ to ${ }^{a} x \in X$. In order to understand how the action core ${ }^{\diamond} \xi: A \diamond X \rightarrow X$ looks, we first need to make explicit what the inclusion $i_{A, X}: A \diamond X \rightarrow A b X$ does. It is easy to see that $A \diamond X$ is the subgroup of $A+X$ generated by the commutators, that is the words of the form $a x a^{-1} x^{-1}$ with $a \in A$ and $x \in X$. The morphism $i_{A, X}$ sends a generator $a x a^{-1} x^{-1}$ to $\left(a x a^{-1}\right)\left(1 x^{-1} 1^{-1}\right)$. This means that the action core ${ }^{\diamond} \xi$ sends an element of the form $a x a^{-1} x^{-1}$ to $\xi\left(\left(a x a^{-1}\right)\left(1 x^{-1} 1^{-1}\right)\right)={ }^{a} x x^{-1}$.

Our last ingredient is the definition of an internal crossed module in a semi-abelian category $\mathbb{A}$. Internal crossed modules are equivalent to internal categories; the conditions that make this happen were obtained in [24]. In order to have a description which is as simple as possible, we require that $\mathbb{A}$ satisfies the so-called Smith is Huq condition $(\mathrm{SH})$, which means that the Smith/Pedicchio commutator [31] of two internal equivalence relations vanishes if and only if so does the Huq commutator of their associated normal subobjects $[1,28]$. Examples of semi-abelian categories that satisfy (SH) include the categories of groups, (commutative) rings (not necessarily unitary), Lie algebras over a commutative ring with unit, Poisson algebras and associative algebras, as are all varieties of such algebras, and crossed modules over those. In fact, all Orzech categories of interest $[30,10]$ are examples. On the other hand, the category of loops is semi-abelian but does not satisfy ( $\mathrm{SH})$.

We now recall the description of internal crossed modules given in $[\mathbf{2 4 , 2 8}$ in the context of a semi-abelian category satisfying (SH). Further details on this definition (and on its general version which does not require $(\mathrm{SH})$ ) can be found in $[\mathbf{2 4}, \mathbf{1 9}, \mathbf{2 8}$ ]. Let us just add here that the crossed module conditions may be expressed in terms of action cores, and that when $(\mathrm{SH})$ does not hold, this approach involves an extra condition in terms of the ternary cosmash product.

Definition 2.24. In a semi-abelian category $\mathbb{A}$ with $(\mathrm{SH})$, an internal crossed module is a pair $(X \stackrel{\partial}{\rightarrow} A, \xi)$ where $\partial: X \rightarrow A$ is a morphism in $\mathbb{A}$ and $\xi: A b X \rightarrow X$ is an internal action such that the diagram

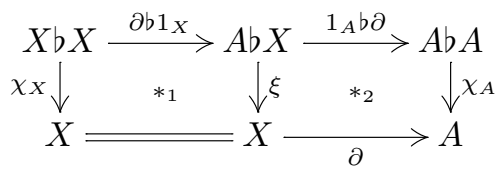

commutes. $*_{1}$ is the Peiffer condition, and $*_{2}$ the precrossed module condition.

\section{Compatible actions of groups}

Definition 3.1. Consider two groups $M$ and $N$ acting on each other via

$$
\xi_{N}^{M}: M b N \rightarrow N \quad \text { and } \quad \xi_{M}^{N}: N b M \rightarrow M
$$


and on themselves by conjugation. We are able to define induced actions $\xi_{M}^{M+N}$ and $\xi_{N}^{M+N}$ of the coproduct $M+N$ on $M$ and on $N$, in such a way that the following diagrams commute:

$$
\begin{aligned}
& \begin{aligned}
M b N \underbrace{\stackrel{i_{M} b 1_{N}}{\longrightarrow}}_{\xi_{N}^{M}}(M+N) b N \\
\downarrow_{N} \xi_{N}^{M+N}
\end{aligned} \\
& \begin{aligned}
N b M & \stackrel{i_{N} b 1_{M}}{\longrightarrow}(M+N) b M \\
\xi_{M}^{N} & \downarrow_{M} \xi_{M}^{M+N}
\end{aligned}
\end{aligned}
$$

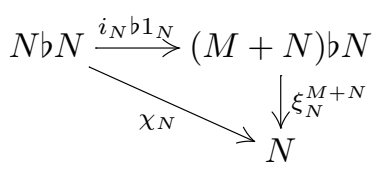

$$
\begin{aligned}
& \begin{array}{r}
M b M \stackrel{i_{M} b 1_{M}}{\longrightarrow}(M+N) b M \\
\chi_{M} \underset{M}{\downarrow_{M}^{\xi_{M}^{M+N}}}
\end{array}
\end{aligned}
$$

This is done by defining the action $\xi_{M}^{M+N}:(M+N) b M \rightarrow M$ on the generators $s \bar{m} s^{-1}$ where $\bar{m} \in M$ and $s \in M+N$, inductively on the length of $s$ :

$$
\xi_{M}^{M+N}\left(s \bar{m} s^{-1}\right)= \begin{cases}\bar{m} & \text { if } s \text { is the empty word, } \\ \xi_{M}^{M+N}\left(s^{\prime} \xi_{M}^{N}\left(n \bar{m} n^{-1}\right) s^{\prime-1}\right) & \text { if } s=s^{\prime} n \text { with } n \in N, \\ \xi_{M}^{M+N}\left(s^{\prime} \chi_{M}\left(m \bar{m} m^{-1}\right) s^{-1}\right) & \text { if } s=s^{\prime} m \text { with } m \in M\end{cases}
$$

and similarly for $\xi_{N}^{M+N}$.

Remark 3.2. In particular, we have that the equalities

$$
\begin{aligned}
& { }^{\left({ }^{n} m\right)} m^{\prime}=\left({ }^{n} m\right) m^{\prime}\left({ }^{n} m\right)^{-1}={ }^{n}\left(m\left({ }^{n^{-1}} m^{\prime}\right) m^{-1}\right)={ }^{n m n^{-1}} m^{\prime}, \\
& \left.{ }^{(m} n\right) n^{\prime}=\left({ }^{m} n\right) n^{\prime}\left({ }^{m} n\right)^{-1}={ }^{m}\left(n\left({ }^{m^{-1}} n^{\prime}\right) n^{-1}\right)={ }^{m n m^{-1}} n^{\prime},
\end{aligned}
$$

where the right-hand sides are given by the induced action of the coproduct, always hold. Diagrammatically this is expressed by the commutativity of

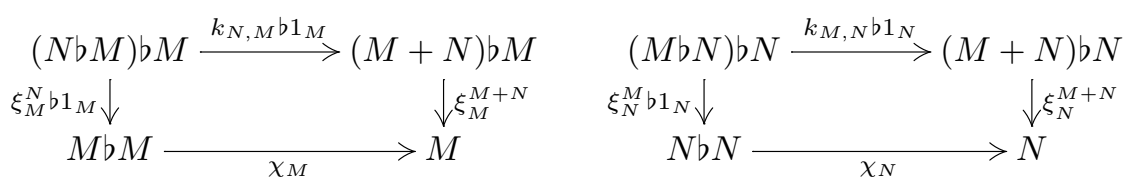

Definition 3.3. Two actions are said to be compatible if also the equalities

$$
\left.{ }^{(m} n\right) m^{\prime}={ }^{m n m^{-1}} m^{\prime} \quad \text { and } \quad\left({ }^{n} m\right) n^{\prime}={ }^{n m n^{-1}} n^{\prime}
$$

hold for each $m, m^{\prime} \in M$ and $n, n^{\prime} \in N$. If once again we examine these equalities from a diagrammatic point of view, then we see that they are equivalent to the commutativity of the diagrams

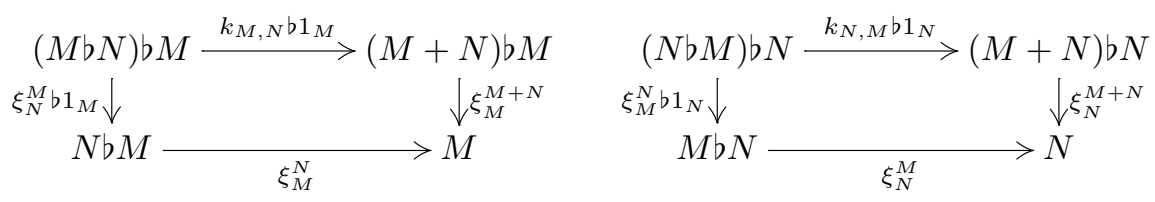

A second look at these four equalities leads us to the following remark.

Remark 3.4. The meaning of (5) and (6) is that for each $m \in M$ and $n \in N$ 
- $\left({ }^{n} m\right) n m^{-1} n^{-1}$ acts trivially on $M$,

- $\left.{ }^{m} n\right) m n^{-1} m^{-1}$ acts trivially on $N$;

whereas the meaning (8) is that for each $m \in M$ and $n \in N$

- $\left({ }^{n} m\right) n m^{-1} n^{-1}$ acts trivially on $N$;

- $\left({ }^{m} n\right) m n^{-1} m^{-1}$ acts trivially on $M$.

If we define $K \leqslant M+N$ to be the normal closure of the subgroup generated by the elements of the form $\left({ }^{n} m\right) n m^{-1} n^{-1}$ or $\left({ }^{m} n\right) m n^{-1} m^{-1}$, we have that $K$ acts trivially on both $M$ and $N$ if and only if the two actions are compatible.

The previous remark leads to the following definition given in [16].

Definition 3.5. Given a pair of compatible actions as above, we define their Peiffer product $M \bowtie N$ of $M$ and $N$ as the quotient

$$
K \triangleright M+N \stackrel{q_{K}}{\longrightarrow} \frac{M+N}{K}-: M \bowtie N .
$$

Remark 3.6. Notice that the morphism $q_{K}$ and the Peiffer product $M \bowtie N$ can equivalently be defined as the coequaliser in the diagram

$$
(N b M)+(M b N) \stackrel{\left(\begin{array}{l}
k_{N, M} \\
k_{M, N}
\end{array}\right)}{\underset{\xi_{M}^{N}+\xi_{N}^{M}}{\longrightarrow}} M+N \stackrel{q}{\longrightarrow} M \bowtie N .
$$

In order to explain why this definition is equivalent to the previous one, consider the morphism $q_{K}$ given by the first definition. It is easy to show that

$$
\left\{\begin{array}{l}
q_{K} \circ i_{M} \circ \xi_{M}^{N}=q_{K} \circ k_{N, M}, \\
q_{K} \circ i_{N} \circ \xi_{N}^{M}=q_{K} \circ k_{M, N}
\end{array}\right.
$$

since this is exactly what taking the quotient by $K$ means. But this is the same as saying

$$
\left\{\begin{array}{l}
q_{K} \circ\left(\xi_{M}^{N}+\xi_{N}^{M}\right) \circ i_{N b M}=q_{K} \circ k_{N, M}, \\
q_{K} \circ\left(\xi_{M}^{N}+\xi_{N}^{M}\right) \circ i_{M b N}=q_{K} \circ k_{M, N}
\end{array}\right.
$$

which in turn is $q_{K} \circ\left(\xi_{M}^{N}+\xi_{N}^{M}\right)=q_{K} \circ\left(\begin{array}{l}k_{N, M} \\ k_{M, N}\end{array}\right)$. The universal property of the coequaliser is given by the universal property of the quotient by $K$ in a straightforward manner.

Given a pair of compatible actions, let $K \leqslant M+N$ be the normal subgroup of Remark 3.4. Since $K$ acts trivially on both $M$ and $N$ we can define induced actions $\xi_{M}^{M \bowtie N}$ and $\xi_{N}^{M \bowtie N}$ of $M \bowtie N$ on $M$ and $N$. They are such that the diagrams

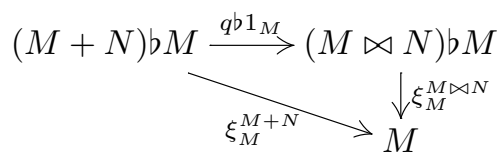

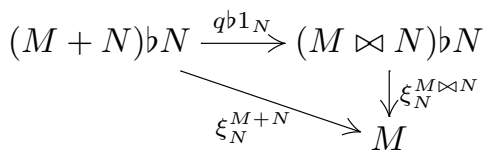

commute. We can describe these actions of the Peiffer product through its universal property, but in order to do so, we need a preliminary remark. 
Remark 3.7. The diagram

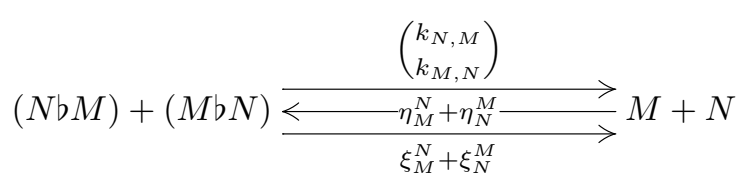

is a reflexive graph. Indeed, the composites $\left(\begin{array}{c}k_{N, M} \\ k_{M, N}\end{array}\right) \circ\left(\eta_{M}^{N}+\eta_{N}^{M}\right)$ and $\left(\xi_{M}^{N}+\xi_{N}^{M}\right) \circ$ $\left(\eta_{M}^{N}+\eta_{N}^{M}\right)$ are equal to $1_{M+N}$ : one is obvious and the other one is clear once we draw the diagram involved.

Lemma 2.9 implies that $q b 1_{M}$ is the coequaliser of $\left(\begin{array}{l}k_{N, M} \\ k_{M, N}\end{array}\right) b 1_{M}$ and $\left(\xi_{M}^{N}+\xi_{N}^{M}\right) b 1_{M}$ and that $q b 1_{N}$ is the coequaliser of $\left(\begin{array}{l}k_{N, M} \\ k_{M, N}\end{array}\right) b 1_{N}$ and $\left(\xi_{M}^{N}+\xi_{N}^{M}\right) b 1_{N}$. We want to use these universal properties to define induced actions $\xi_{M}^{M \bowtie N}$ and $\xi_{N}^{M \bowtie N}$ of $M \bowtie N$ on $M$ and $N$ as in Figure 1. In order to do so, we need the next result.

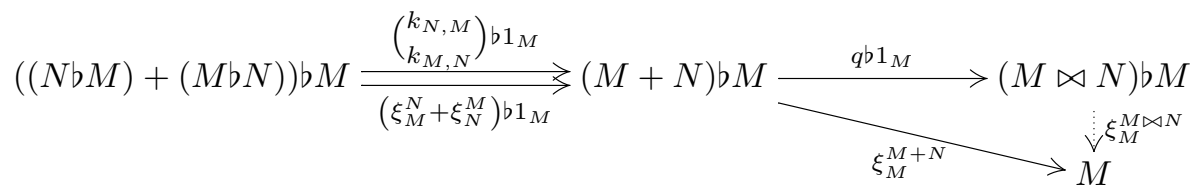

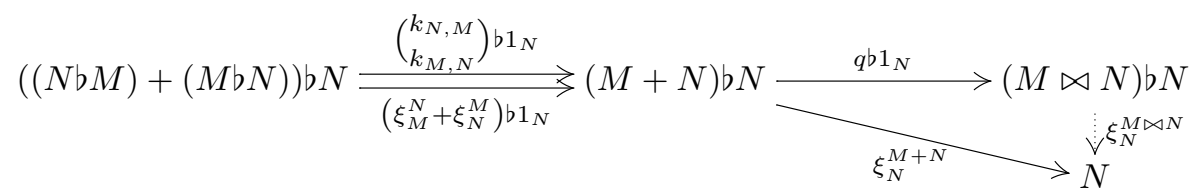

Figure 1: Induced actions of the Peiffer product.

Proposition 3.8. The action $\xi_{M}^{M+N}$ coequalises $\left(\begin{array}{l}k_{N, M} \\ k_{M, N}\end{array}\right) b 1_{M}$ and $\left(\xi_{M}^{N}+\xi_{N}^{M}\right) b 1_{M}$. Similarly, the action $\xi_{N}^{M+N}$ coequalises $\left(\begin{array}{l}k_{N, M} \\ k_{M, N}\end{array}\right) b 1_{N}$ and $\left(\xi_{M}^{N}+\xi_{N}^{M}\right) b 1_{N}$.

Proof. Consider a generator $s \bar{m} s^{-1}$ of $((N b M)+(M b N)) b M$ and write $s$ as juxtaposition of generators of $N b M$ and $M b N$, that is $s=s_{1} \cdots s_{k}$ with $s_{j}=n_{j} m_{j} n_{j}^{-1} \in$ $N b M$ or $s_{j}=m_{j} n_{j} m_{j}^{-1} \in M b N$. We are going to prove the equality

$$
\xi_{M}^{M+N}\left(\left(\left(\begin{array}{l}
k_{N, M} \\
k_{M, N}
\end{array}\right) b 1_{M}\right)\left(s \bar{m} s^{-1}\right)\right)=\xi_{M}^{M+N}\left(\left(\left(\xi_{M}^{N}+\xi_{N}^{M}\right) b 1_{M}\right)\left(s \bar{m} s^{-1}\right)\right)
$$

by induction on $k$. First of all, notice that it is equivalent to the equality

$$
\xi_{M}^{M+N}\left(s \bar{m} s^{-1}\right)=\xi_{M}^{M+N}\left(\epsilon(s) \bar{m} \epsilon(s)^{-1}\right),
$$

where $\epsilon(s):-\left(\xi_{M}^{N}+\xi_{N}^{M}\right)(s) \in M+N$. In order to prove it when $s$ is the empty word, it suffices to notice that also $\epsilon(s)$ is the empty word. Now suppose we proved (12) for each word whose decomposition involves at most $k-1$ generators of $N b M$ and $M b N$, 
consider $s=s_{1} \cdots s_{k}$ and denote $s^{\prime}=s_{1} \cdots s_{k-1}$ : we have the chain of equalities

$$
\begin{aligned}
\xi_{M}^{M+N}\left(s \bar{m} s^{-1}\right) & =\xi_{M}^{M+N}\left(s^{\prime} s_{k} \bar{m} s_{k}^{-1} s^{\prime-1}\right)=\xi_{M}^{M+N}\left(s^{\prime}\left(s_{k} \bar{m}\right) s^{\prime-1}\right) \\
& =\xi_{M}^{M+N}\left(s^{\prime}\left({ }^{\epsilon\left(s_{k}\right)} \bar{m}\right) s^{\prime-1}\right)=\xi_{M}^{M+N}\left(\epsilon\left(s^{\prime}\right)\left({ }^{\epsilon\left(s_{k}\right)} \bar{m}\right) \epsilon\left(s^{\prime}\right)^{-1}\right) \\
& =\xi_{M}^{M+N}\left(\epsilon\left(s^{\prime}\right) \epsilon\left(s_{k}\right) \bar{m} \epsilon\left(s_{k}\right)^{-1} \epsilon\left(s^{\prime}\right)^{-1}\right)=\xi_{M}^{M+N}\left(\epsilon(s) \bar{m} \epsilon(s)^{-1}\right),
\end{aligned}
$$

where

$$
\epsilon\left(s_{k}\right)= \begin{cases}{ }^{n_{k}} m_{k} & \text { if } s_{k}=n_{k} m_{k} n_{k}^{-1} \in N b M, \\ { }^{m_{k}} n_{k} & \text { if } s_{k}=m_{k} n_{k} m_{k}^{-1} \in M b N .\end{cases}
$$

Finally, we apply the same reasoning to $\xi_{N}^{M+N}$.

Proposition 3.9. We have two crossed module structures

$$
\left(M \stackrel{l_{M}}{\longrightarrow} M \bowtie N, \xi_{M}^{M \bowtie N}\right), \quad\left(N \stackrel{l_{N}}{\longrightarrow} M \bowtie N, \xi_{N}^{M \bowtie N}\right),
$$

where the actions of the Peiffer product are induced as above and the morphisms $l_{M}$ and $l_{N}$ are defined through

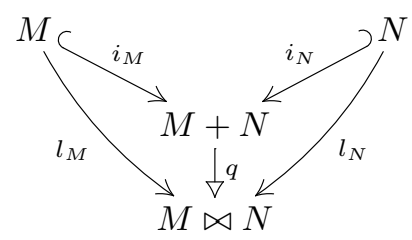

Proof. We will show the claim only for $\xi_{M}^{M \bowtie N}$, since the proof in the other case uses the same strategy. We need to show the commutativity of the following squares

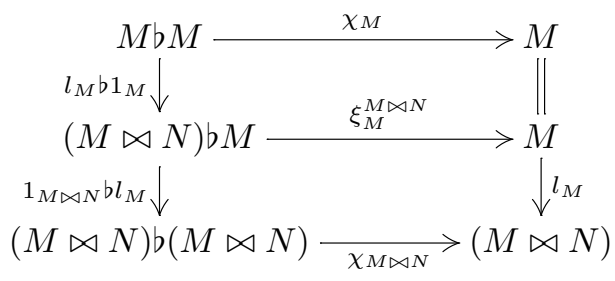

For the commutativity of the upper square we have the chain of equalities

$$
\xi_{M}^{M \bowtie N} \circ\left(l_{M} b 1_{M}\right)=\xi_{M}^{M \bowtie N} \circ\left(q b 1_{M}\right) \circ\left(i_{M} b 1_{M}\right)=\xi_{M}^{M+N} \circ\left(i_{M} b 1_{M}\right)=\chi_{M}
$$

given by commutativity of diagrams (3) and (11).

For what concerns the lower square, it can be shown to be commutative by direct calculations, using the explicit definition of the coproduct action given in (4). First of all we can precompose with the regular epimorphism $q b 1_{M}$ : we find

$$
l_{M} \circ \xi_{M}^{M \bowtie N} \circ\left(q b 1_{M}\right)=q \circ i_{M} \circ \xi_{M}^{M+N}
$$

by (13) and the top commutative triangle in Figure 1, while

$$
\chi_{M \bowtie N} \circ\left(1_{M \bowtie N} b l_{M}\right) \circ\left(q b 1_{M}\right)=q \circ \chi_{M+N} \circ\left(1_{M+N} b i_{M}\right)
$$

follows from (13) and the equivariance of $q$ with respect to the conjugation actions 
$\chi_{M+N}$ and $\chi_{M \bowtie N}$. Hence the required commutativity is equivalent to the equation

$$
q \circ \chi_{M+N} \circ\left(1_{M+N} b i_{M}\right)=q \circ i_{M} \circ \xi_{M}^{M+N} .
$$

Now we can take a word $s \in M+N$, an element $\bar{m} \in M$. We observe that the two morphisms in (14) send the general element $s \bar{m} s^{-1} \in(M+N) b M$ to $q\left({ }^{s} \bar{m}\right)$ and $q\left(s \bar{m} s^{-1}\right)$, respectively. We prove the equality

$$
q\left({ }^{s} \bar{m}\right)=q\left(s \bar{m} s^{-1}\right)
$$

by induction on the length of $s$. First let $s$ have length 0 , so that it is the empty word: we have that ${ }^{s} \bar{m}=\bar{m}=s \bar{m} s^{-1}$ and hence (15). For the induction step we are going to use the equality $q\left({ }^{n} \bar{m}\right)=q\left(n \bar{m} n^{-1}\right)$ coming from the definition of the Peiffer product. Suppose that (15) holds for words $s$ with length $l(s)<k$. Given $s$ with length $k$ we can write it as $s=x s^{\prime}$ with $x=m \in M$ or $x=n \in N$ and $l\left(s^{\prime}\right)=k-1$ : now we have the chain of equalities

$$
\begin{aligned}
q\left({ }^{s} \bar{m}\right) & =q\left({ }^{x s^{\prime}} \bar{m}\right)=q\left({ }^{x}\left(s^{\prime} \bar{m}\right)\right)=q\left(x\left(s^{\prime} \bar{m}\right) x^{-1}\right)=q(x) q\left({ }^{\prime} \bar{m}\right) q\left(x^{-1}\right) \\
& =q(x) q\left(s^{\prime} \bar{m} s^{\prime-1}\right) q\left(x^{-1}\right)=q\left(x s^{\prime} \bar{m} s^{-1} x^{-1}\right)=q\left(s \bar{m} s^{-1}\right) .
\end{aligned}
$$

We conclude that $\left(M \stackrel{l_{M}}{\longrightarrow} M \bowtie N, \xi_{M}^{M \bowtie N}\right)$ and $\left(N \stackrel{l_{N}}{\longrightarrow} M \bowtie N, \xi_{N}^{M \bowtie N}\right)$ are crossed modules.

Furthermore we know that the actions $\xi_{N}^{M}$ and $\xi_{M}^{N}$ are in turn induced by $\xi_{M}^{M \bowtie N}$ and $\xi_{N}^{M \bowtie N}$ through the morphisms $l_{M}$ and $l_{N}$, that is
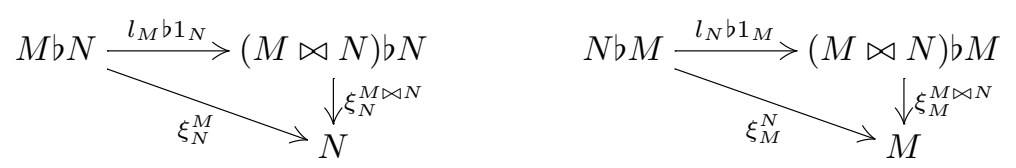

commute. This can be proved by using diagrams (2), (3), (11) and (13).

Proposition 3.10 (Remark 2.16 in [6]). Two actions as above are compatible if and only if there exists a group $L$ and two crossed module structure $\left(M \stackrel{\mu}{\rightarrow} L, \xi_{M}^{L}\right)$ and $\left(N \stackrel{\nu}{\rightarrow} L, \xi_{N}^{L}\right)$ such that the actions of $M$ on $N$ and the action of $N$ on $M$ are induced from $L$ and its actions.

Proof. $(\Leftarrow)$ We first show that the actions $\xi_{N}^{M}:-\xi_{N}^{L} \circ\left(\mu b 1_{N}\right)$ and $\xi_{M}^{N}:-\xi_{M}^{L} \circ\left(\nu b 1_{M}\right)$ are compatible. To see that they are actually actions it suffices to use Remark 2.21. In order to prove (8) - we will show only one of the two equalities, since the proof of the other follows the same steps - we are going to use the commutative diagrams induced from the crossed module structures involving $L$, that is
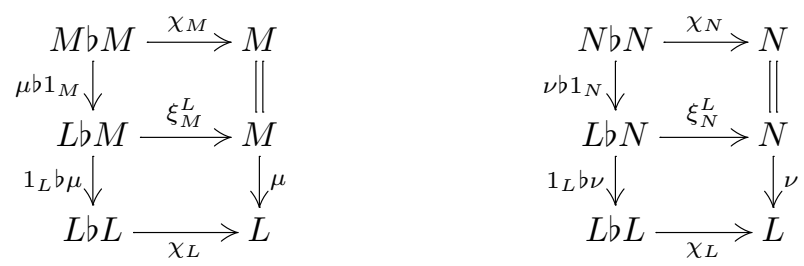
This gives us the chain of equalities

$$
\begin{aligned}
{ }^{\left({ }^{m} n\right)} m^{\prime} & ={ }^{\nu\left({ }^{\mu(m)} n\right)} m^{\prime}={ }^{\mu(m) \nu(n) \mu\left(m^{-1}\right)} m^{\prime}={ }^{\mu(m) \nu(n)}\left({ }^{\mu\left(m^{-1}\right)} m^{\prime}\right) \\
& ={ }^{\mu(m) \nu(n)}\left({ }^{m^{-1}} m^{\prime}\right)={ }^{\mu(m)}\left({ }^{\nu(n)}\left({ }^{m^{-1}} m^{\prime}\right)\right)={ }^{\mu(m)}\left({ }^{n}\left({ }^{m^{-1}} m^{\prime}\right)\right) \\
& ={ }^{\mu(m)}\left({ }^{n m^{-1}} m^{\prime}\right)={ }^{m}\left({ }^{n m^{-1}} m^{\prime}\right)={ }^{m n m^{-1}} m^{\prime} .
\end{aligned}
$$

$(\Rightarrow)$ This implication is given by Proposition 3.9.

\section{Compatible actions in semi-abelian categories}

From now on we will consider $\mathbb{A}$ to be a semi-abelian category in which the condition (SH) holds.

We are going to give a definition of compatible internal actions which is inspired by Definitions 3.1 and 3.3, with some differences that we will explain here.
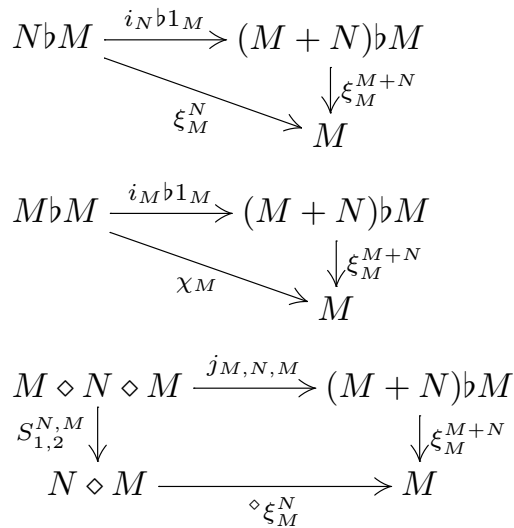
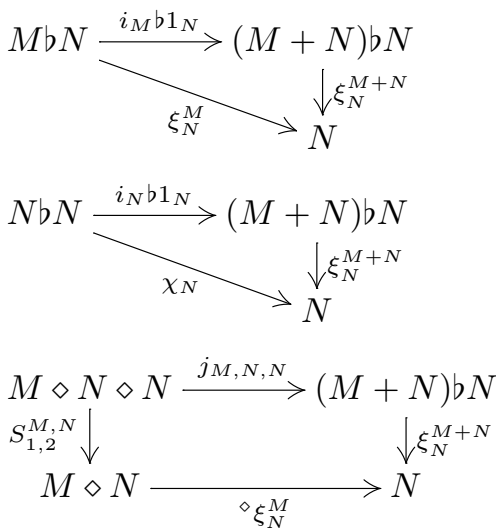

Figure 2: The diagrams (CA.0).

Definition 4.1. Consider two objects $M, N \in \mathbb{A}$ which act on each other and on themselves by conjugation and denote the actions as

$$
\begin{array}{ll}
\chi_{M}: M b M \rightarrow M, & \chi_{N}: N b N \rightarrow N, \\
\xi_{N}^{M}: M b N \rightarrow N, & \xi_{M}^{N}: N b M \rightarrow M .
\end{array}
$$

We say that the actions $\xi_{N}^{M}$ and $\xi_{M}^{N}$ are compatible if there exist two actions

$$
\xi_{N}^{M+N}:(M+N) b N \rightarrow N, \quad \xi_{M}^{M+N}:(M+N) b M \rightarrow M
$$

"induced" from $\xi_{N}^{M}, \xi_{M}^{N}$ and the conjugations, that is such that the diagrams (CA.0) in Figure 2 as well as the diagrams (CA.M) and (CA.N) in Figure 3 commute.

This definition obviously implies the one given in the case of groups, but we will see later (Corollary 4.12) that in Grp the two definitions coincide. The difference between these two definitions is twofold. 


$$
\begin{aligned}
& ((N b M)+(M b N)) b M \stackrel{\left(\xi_{M}^{N}+\xi_{N}^{M}\right) b 1_{M}}{\longrightarrow}(M+N) b M
\end{aligned}
$$

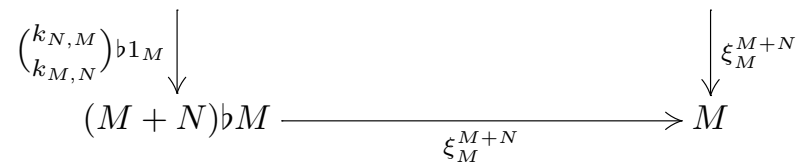

$$
\begin{aligned}
& ((N b M)+(M b N)) b N \stackrel{\left(\xi_{M}^{N}+\xi_{N}^{M}\right) b 1_{N}}{\longrightarrow}(M+N) b N
\end{aligned}
$$

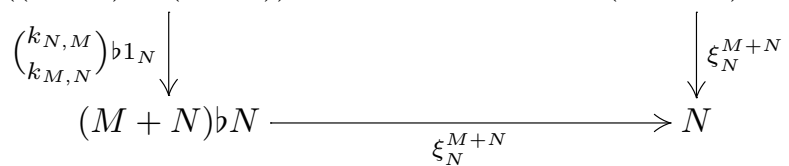

Figure 3: The diagrams (CA.M) and (CA.N).

- First of all, the commutativity of the two squares in (CA.0) involving the ternary cosmash products is for free in Grp (and in $\mathbf{L i e} \mathbf{H}_{R}$ as one can see from [13]). Right now it is not clear to us what are the conditions the category $\mathbb{A}$ must satisfy for the commutativity of these squares to be implied by the other four triangles in (CA.0). Note that this is quite similar to the ternary cosmash product conditions that appear in the description of internal crossed modules given in [19].

- Likewise, note the difference between diagrams (CA.M) and (CA.N), and their version for groups given by (9). The former two can be decomposed into the latter, together with (7) and with additional conditions involving $b$ and higherorder cosmash products. Also this aspect would benefit from further investigation.

Remark 4.2. Notice that in the situation of the previous definition, the coproduct actions $\xi_{M}^{M+N}$ and $\xi_{N}^{M+N}$ are uniquely determined by the commutativities of (CA.0) due to Lemma 2.13.

Proposition 4.3. Given a pair of coterminal crossed modules

$$
\left(M \stackrel{\mu}{\rightarrow} L, \xi_{M}^{L}\right), \quad\left(N \stackrel{\nu}{\rightarrow} L, \xi_{N}^{L}\right)
$$

we can define actions $\xi_{N}^{M}$ and $\xi_{M}^{N}$ through the diagrams
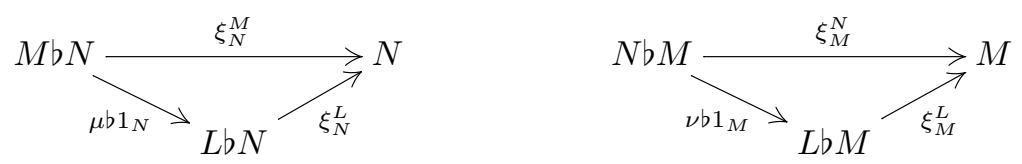

These actions are then compatible in the sense of Definition 4.1.

Proof. First of all, notice that $\xi_{N}^{M}$ and $\xi_{M}^{N}$ are actually actions due to Remark 2.21. Now, in order to show that they are compatible, we need to define the coproduct 
actions

$$
\xi_{N}^{M+N}:(M+N) b N \rightarrow N, \quad \xi_{M}^{M+N}:(M+N) b M \rightarrow M,
$$

such that diagrams (CA.0), (CA.M) and (CA.N) commute. These are defined as the compositions
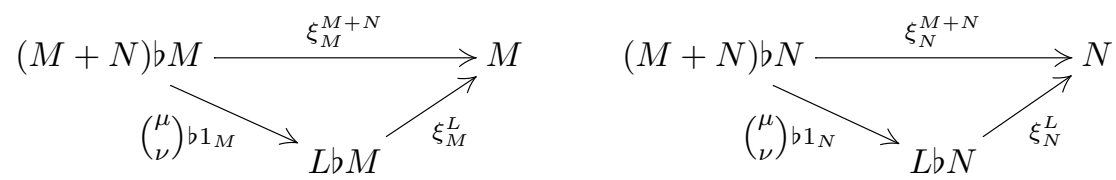

Once again the fact that they are actions is given by Remark 2.21. In order to show that the four triangles in (CA.0) commute, we simply calculate

$$
\begin{aligned}
& \xi_{M}^{M+N} \circ\left(i_{M} b 1_{M}\right)=\xi_{M}^{L} \circ\left(\left(\begin{array}{l}
\mu \\
\nu
\end{array}\right) b 1_{M}\right) \circ\left(i_{M} b 1_{M}\right)=\xi_{M}^{L} \circ\left(\mu b 1_{M}\right)=\chi_{M}, \\
& \xi_{M}^{M+N} \circ\left(i_{N} b 1_{M}\right)=\xi_{M}^{L} \circ\left(\left(\begin{array}{l}
\mu \\
\nu
\end{array}\right) b 1_{M}\right) \circ\left(i_{N} b 1_{M}\right)=\xi_{M}^{L} \circ\left(\nu b 1_{M}\right)=\xi_{M}^{N}, \\
& \xi_{N}^{M+N} \circ\left(i_{M} b 1_{N}\right)=\xi_{N}^{L} \circ\left(\left(\begin{array}{c}
\mu \\
\nu
\end{array}\right) b 1_{N}\right) \circ\left(i_{M} b 1_{N}\right)=\xi_{N}^{L} \circ\left(\mu b 1_{N}\right)=\xi_{N}^{M}, \\
& \xi_{N}^{M+N} \circ\left(i_{N} b 1_{N}\right)=\xi_{N}^{L} \circ\left(\left(\begin{array}{c}
\mu \\
\nu
\end{array}\right) b 1_{N}\right) \circ\left(i_{N} b 1_{N}\right)=\xi_{N}^{L} \circ\left(\nu b 1_{N}\right)=\chi_{N}
\end{aligned}
$$

using the crossed module conditions. For the first square in (CA.0), we use the diagrams
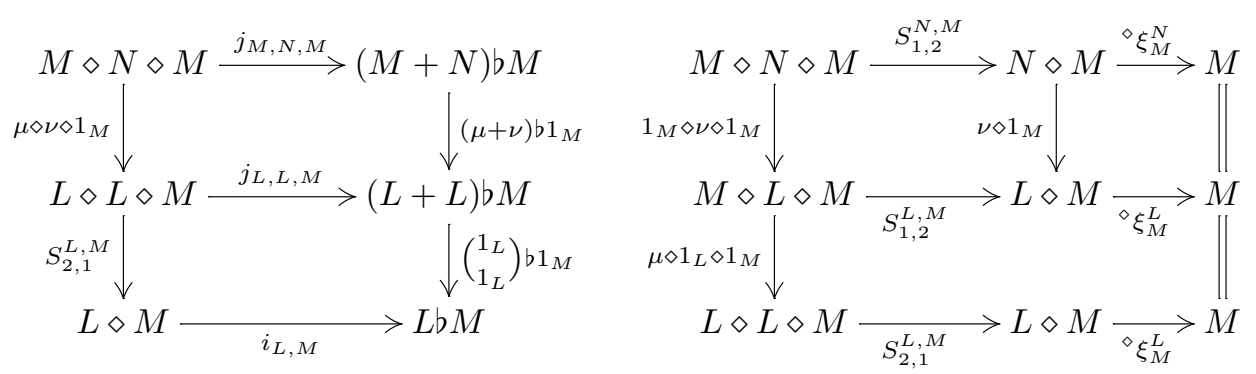

in order to obtain the chain of equalities

$$
\begin{aligned}
\xi_{M}^{M+N} \circ j_{M, N, M} & =\xi_{M}^{L} \circ\left(\left(\begin{array}{l}
\mu \\
\nu
\end{array}\right) b 1_{M}\right) \circ j_{M, N, M}=\xi_{M}^{L} \circ i_{L, M} \circ S_{2,1}^{L, M} \circ\left(\mu \diamond \nu \diamond 1_{M}\right) \\
& ={ }^{\diamond} \xi_{M}^{N} \circ S_{1,2}^{N, M} .
\end{aligned}
$$

These diagrams are induced by naturality and by the crossed module conditions. For the one on the right, we may use Theorem 5.6 in [19], which (modulo the notation $\otimes$ for $\diamond$, together with $\left({ }^{\diamond} \xi_{M}^{L}\right)_{1,2}$ for ${ }^{\diamond} \xi_{M}^{L} \circ S_{1,2}^{L, M}$ and $\left({ }^{\diamond} \xi_{M}^{L}\right)_{2,1}$ for $\left.{ }^{\diamond} \xi_{M}^{L} \circ S_{2,1}^{L, M}\right)$ tells us that the diagram

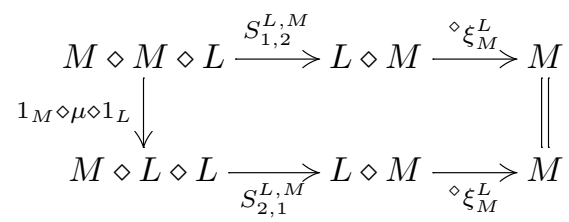

commutes: this is precisely the bottom rectangle in the right hand side diagram above, 
composed with the canonical isomorphism induced by the symmetry in the co-smash products.

Through a similar reasoning we may prove the commutativity of the other square in (CA.0). Finally, we need to show (CA.M), that is the fact that $\xi_{M}^{M+N}$ coequalises the morphisms

$$
((N b M)+(M b N)) b M \frac{\left(\begin{array}{l}
k_{N, M} \\
k_{M, N}
\end{array}\right) b 1_{M}}{\left(\xi_{M}^{N}+\xi_{N}^{M}\right) b 1_{M}} \rightarrow(M+N) b M .
$$

Here we have the chain of equalities

$$
\begin{aligned}
& \xi_{M}^{M+N} \circ\left(\xi_{M}^{N}+\xi_{N}^{M}\right) b 1_{M}=\xi_{M}^{L} \circ\left(\begin{array}{c}
\mu \\
\nu
\end{array}\right) b 1_{M} \circ\left(\left(\left(\xi_{M}^{L} \circ \nu b 1_{M}\right)+\left(\xi_{N}^{L} \circ \mu b 1_{N}\right)\right) b 1_{M}\right) \\
& =\xi_{M}^{L} \circ\left(\left(\left(\begin{array}{c}
\mu \\
\nu
\end{array}\right) \circ\left(\left(\xi_{M}^{L} \circ \nu b 1_{M}\right)+\left(\xi_{N}^{L} \circ \mu b 1_{N}\right)\right)\right) b 1_{M}\right) \\
& =\xi_{M}^{L} \circ\left(\begin{array}{c}
\mu \circ \xi_{M}^{L} \circ \nu b 1_{M} \\
\nu \circ \xi_{N}^{L} \circ \mu b 1_{N}
\end{array}\right) b 1_{M} \\
& =\xi_{M}^{L} \circ\left(\begin{array}{c}
\chi_{L} \circ \nu b \mu \\
\chi_{L} \circ \mu b \nu
\end{array}\right) b 1_{M}
\end{aligned}
$$

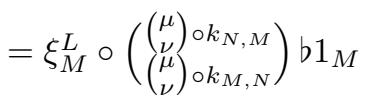

$$
\begin{aligned}
& =\xi_{M}^{L} \circ\left(\left(\begin{array}{c}
\mu \\
\nu
\end{array}\right) b 1_{M}\right) \circ\left(\begin{array}{l}
k_{N, M} \\
k_{M, N}
\end{array}\right) b 1_{M} \\
& =\xi_{M}^{M+N} \circ\left(\left(\begin{array}{l}
k_{N, M} \\
k_{M, N}
\end{array}\right) b 1_{M}\right) \text {. }
\end{aligned}
$$

Through a similar reasoning we can show that (CA.N) commutes.

We take the construction of the Peiffer product in (10) as a general definition.

Definition 4.4. Given two objects $M$ and $N$ acting on each other via $\xi_{M}^{N}$ and $\xi_{N}^{M}$, we define their Peiffer product $M \bowtie N$ as the coequaliser

$$
(N b M)+(M b N) \underset{\xi_{M}^{N}+\xi_{N}^{M}}{\stackrel{\left(\begin{array}{l}
k_{N, M} \\
k_{M, N}
\end{array}\right)}{\longrightarrow}} M+N \stackrel{q \longrightarrow}{\longrightarrow} M \bowtie N .
$$

An equivalent definition of the Peiffer product of two actions can be given through the following proposition, which characterises it as the pushout of the two semi-direct products induced by the two actions.

Proposition 4.5. Given a pair of actions $\xi_{N}^{M}: M b N \rightarrow N$ and $\xi_{M}^{N}: N b M \rightarrow M$ we can obtain the Peiffer product $M \bowtie N$ as the pushout

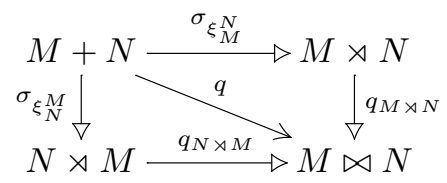

of the two semi-direct products. 
Proof. Recall that the semi-direct products are defined as the coequalisers

$$
\begin{aligned}
& N b M \underset{k_{N, M}}{\stackrel{i_{M} \circ \xi_{M}^{N}}{\longrightarrow}} M+N \stackrel{\sigma_{\xi_{M}^{N}}}{\longrightarrow} M \rtimes N,
\end{aligned}
$$

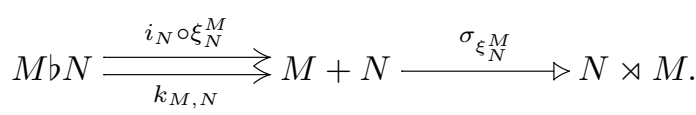

By definition we know that $q$ coequalises each of these pairs of morphisms, and thus we obtain the unique regular epimorphisms $q_{N \rtimes M}$ and $q_{M \rtimes N}$ making the triangles

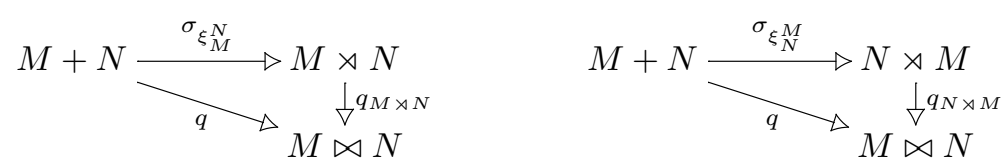

commute. To prove that (17) is a pushout, suppose there exist $f: M \rtimes N \rightarrow Z$ and $g: N \rtimes M \rightarrow Z$ such that $\gamma:-f \circ \sigma_{\xi_{M}^{N}}=g \circ \sigma_{\xi_{N}^{M}}$. It suffices to prove that $\gamma$ coequalises the morphisms defining $q$ :

$\gamma \circ\left(\begin{array}{l}k_{N, M} \\ k_{M, N}\end{array}\right)=\left(\begin{array}{c}\gamma \circ k_{N, M} \\ \gamma \circ k_{M, N}\end{array}\right)=\left(\begin{array}{c}f \circ \sigma_{\xi_{M}^{N}} \circ k_{N, M} \\ g \circ \sigma_{\xi_{N}^{M}} \circ k_{M, N}\end{array}\right)=\left(\begin{array}{c}f \circ \sigma_{\xi_{M}^{N}} \circ i_{M} \circ \xi_{M}^{N} \\ g \circ \sigma_{\xi_{N}^{M}} \circ i_{N} \circ \xi_{N}^{M}\end{array}\right)=\left(\begin{array}{c}\gamma \circ i_{M} \circ \xi_{M}^{N} \\ \gamma \circ i_{N} \circ \xi_{N}^{M}\end{array}\right)=\gamma \circ\left(\xi_{M}^{N}+\xi_{N}^{M}\right)$.

This gives us a unique morphism $\gamma^{\prime}: M \bowtie N \rightarrow Z$ such that $\gamma^{\prime} \circ q_{M \rtimes N}=f$ and $\gamma^{\prime} \circ q_{N \rtimes M}=g$ because $\sigma_{\xi_{M}^{N}}$ and $\sigma_{\xi_{N}^{M}}$ are epimorphisms.

The idea behind the Peiffer product $M \bowtie N$ is that it should be the universal object acting on $M$ and $N$ with two crossed modules structures, as soon as these two objects act on each other compatibly. This is meant to solve the following problem. If we are in the situation of two compatible actions, we have induced coproduct actions whose precrossed module conditions

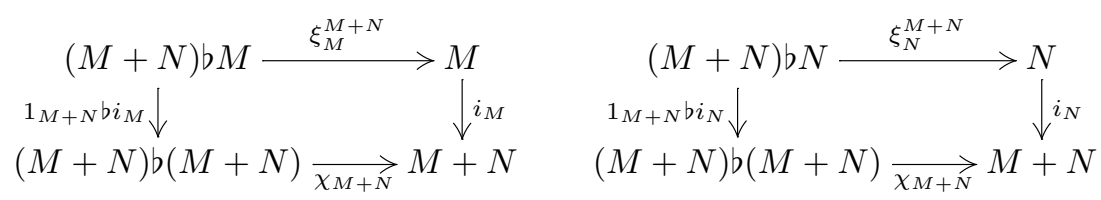

are generally not satisfied. (However, the Peiffer conditions always hold.)

Hence we want to do two things: we want to define actions of the Peiffer product on $M$ and $N$ induced from the coproduct actions, and then we want to show that the postcomposition with the quotient defining the Peiffer product makes the previous squares commute, so that we obtain two crossed module structures.

Again by using Lemma 2.9 and Remark 3.7 we deduce that in order to define the actions $\xi_{M}^{M \bowtie N}$ and $\xi_{N}^{M \bowtie N}$ of the Peiffer product as in Figure 4 (compare with the group case, Figure 1) it suffices to show that $\xi_{M}^{M+N}$ coequalises the parallel morphisms in (19) and that $\xi_{N}^{M+N}$ coequalises the parallel morphisms in (20). These conditions are equivalent to the commutativity of (CA.M) and (CA.N).

Now we have the desired actions of the Peiffer product, but in order to obtain the crossed module structures we need to show that postcomposing with the quotient $q$ makes the diagrams (18) commute. In fact, we are going to prove more than this: the Peiffer product is the coequaliser of those morphisms! 


$$
\begin{aligned}
& ((N b M)+(M b N)) b M \frac{\left(\begin{array}{l}
k_{N, M} \\
k_{M, N}
\end{array}\right) b 1_{M}}{\underset{\left(\xi_{M}^{N}+\xi_{N}^{M}\right) b 1_{M}}{\longrightarrow}}(M+N) b M \stackrel{q b 1_{M}}{\longrightarrow}(M \bowtie N) b M
\end{aligned}
$$

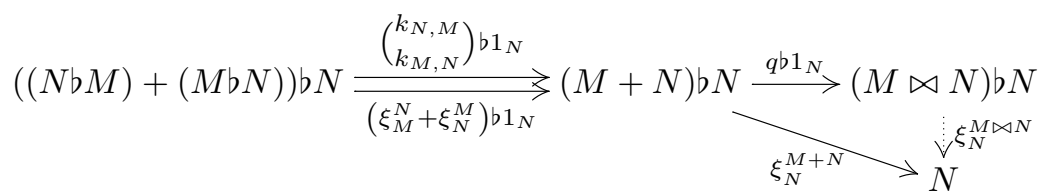

Figure 4: Actions $\xi_{M}^{M \bowtie N}$ and $\xi_{N}^{M \bowtie N}$ of the Peiffer product.

Definition 4.6. Given a pair of compatible actions $\xi_{M}^{N}$ and $\xi_{N}^{M}$, we define the strong Peiffer product $M \bowtie_{S} N$ as the coequaliser in the diagram

$$
((M+N) b M)+((M+N) b N) \frac{\chi_{M+N} \circ\left(\begin{array}{l}
1_{M+N} b i_{M} \\
1_{M+N} b i_{N}
\end{array}\right)}{\xi_{M}^{M+N}+\xi_{N}^{M+N}} M+N \stackrel{q_{S}}{\longrightarrow} M \bowtie_{S} N .
$$

Remark 4.7. It is important to notice that in principle there is a huge difference between the coequaliser in (16) and the one in (21):

- the latter makes sense only if the two actions are already compatible - otherwise the existence of the coproduct actions is not guaranteed; by definition, the strong Peiffer product coequalises the compositions in (18);

- the former makes sense even when the two actions are not compatible; it is obtained following the ideas from the particular compatibility conditions in the case of Grp through Remark 3.4 and Remark 3.6.

This means that by taking (16) as a definition of $M \bowtie N$, we would not immediately have that the Peiffer product is the universal way to coequalise the compositions in (18). Obviously if we precompose the morphisms in (21) with $\left(i_{N} b 1_{M}\right)+\left(i_{M} b 1_{N}\right)$, we see that $q_{S}$ coequalises also the morphisms defining $q$

$$
q_{S} \circ\left(\xi_{M}^{N}+\xi_{N}^{M}\right)=q_{S} \circ\left(\begin{array}{l}
k_{N, M} \\
k_{M, N}
\end{array}\right)
$$

but for the converse we need the following proposition.

Proposition 4.8. Consider two actions $\xi_{N}^{M}$ and $\xi_{M}^{N}$ which are compatible in the sense of Definition 4.1. Then the Peiffer product $M \bowtie N$ as in (16) and the strong Peiffer product $M \bowtie_{S} N$ as in (21) are isomorphic.

Proof. In order to obtain the needed isomorphism it suffices to show that $q$ coequalises the morphisms defining $q_{S}$ : since the converse already holds due to Remark 4.7, we obtain the claim by the universal properties of the coequalisers. Recalling Lemma 2.13 
we just need to show that $q$ coequalises the two compositions in

$$
\begin{gathered}
(M b M)+(N b M)+(M \diamond N \diamond M)+(M b N)+(N b N)+(M \diamond N \diamond N) \\
\left(\begin{array}{c}
i_{M} b 1_{M} \\
i_{N} b 1_{M} \\
j_{M, N, M}
\end{array}\right)+\left.\left(\begin{array}{c}
i_{M} b 1_{N} \\
i_{N} b 1_{N} \\
j_{M, N, N}
\end{array}\right)\right|_{\nabla} \\
((M+N) b M)+((M+N) b N) \frac{\chi_{M+N} \circ\left(\begin{array}{l}
1_{M+N} b i_{M} \\
1_{M+N} b i_{N}
\end{array}\right)}{\xi_{M}^{M+N}+\xi_{N}^{M+N}} M+N
\end{gathered}
$$

By the universal property of the coproduct we can consider each component separately and since the last three are similar to the first three - it suffices to exchange $M$ and $N$-we are going to examine only the first three.

- Precomposing with the inclusion of $M b M$, we obtain

$$
\begin{aligned}
q \circ \chi_{M+N} \circ\left(\begin{array}{c}
1_{M+N} b i_{M} \\
1_{M+N} b i_{N}
\end{array}\right) \circ i_{1} \circ\left(i_{M} b 1_{M}\right) & =q \circ \chi_{M+N} \circ\left(i_{M} b i_{M}\right)=q \circ i_{M} \circ \chi_{M} \\
& =q \circ i_{M} \circ \xi_{M}^{M+N} \circ\left(i_{M} b 1_{M}\right) \\
& =q \circ\left(\xi_{M}^{M+N}+\xi_{N}^{M+N}\right) \circ i_{1} \circ\left(i_{M} b 1_{M}\right) .
\end{aligned}
$$

- Precomposing with the inclusion of $N b M$, and using the definition of $q$ we obtain

$$
\begin{aligned}
q \circ \chi_{M+N} \circ\left(\begin{array}{c}
1_{M+N} b i_{M} \\
1_{M+N} b i_{N}
\end{array}\right) \circ i_{1} \circ\left(i_{N} b 1_{M}\right) & =q \circ \chi_{M+N} \circ\left(i_{N} b i_{M}\right)=q \circ k_{N, M} \\
& =q \circ i_{M} \circ \xi_{M}^{N}=q \circ i_{M} \circ \xi_{M}^{M+N} \circ\left(i_{N} b 1_{M}\right) \\
& =q \circ\left(\xi_{M}^{M+N}+\xi_{N}^{M+N}\right) \circ i_{1} \circ\left(i_{N} b 1_{M}\right) .
\end{aligned}
$$

- Precomposing with the inclusion of $M \diamond N \diamond M$, we obtain

$$
\begin{aligned}
q \circ \chi_{M+N} \circ\left(\begin{array}{c}
1_{M+N} b i_{M} \\
1_{M+N} b i_{N}
\end{array}\right) \circ i_{1} \circ j_{M, N, M} & =q \circ \chi_{M+N} \circ\left(1_{M+N} b i_{M}\right) \circ j_{M, N, M} \\
& =q \circ h_{N, M} \circ S_{1,2}^{N, M}=q \circ k_{N, M} \circ i_{N, M} \circ S_{1,2}^{N, M} \\
& =q \circ i_{M} \circ \xi_{M}^{N} \circ i_{N, M} \circ S_{1,2}^{N, M} \\
& =q \circ i_{M} \circ \xi_{M}^{M+N} \circ j_{M, N, M} \\
& =q \circ\left(\xi_{M}^{M+N}+\xi_{N}^{M+N}\right) \circ i_{1} \circ j_{M, N, M}
\end{aligned}
$$

This means that $M \bowtie_{S} N \cong M \bowtie N$ and that $q$ is the universal morphism making (18) commute through postcomposition.

Our aim now is to show that $\xi_{M}^{M \bowtie N}$ and $\xi_{N}^{M \bowtie N}$ are indeed actions, which moreover, induce two crossed module structures.

Proposition 4.9. The morphisms $\xi_{M}^{M \bowtie N}$ and $\xi_{N}^{M \bowtie N}$ are internal actions. We have crossed module structures

$$
\left(M \stackrel{l_{M}}{\longrightarrow} M \bowtie N, \xi_{M}^{M \bowtie N}\right) \quad\left(N \stackrel{l_{N}}{\longrightarrow} M \bowtie N, \xi_{N}^{M \bowtie N}\right)
$$

where the morphisms $l_{M}$ and $l_{N}$ are defined as in (13). Further, as in Proposition 4.3, the compatible actions induced by these crossed module structures coincide with the actions $\xi_{M}^{N}$ and $\xi_{N}^{M}$.

Proof. We are going to prove the claim only for $\xi_{M}^{M \bowtie N}$ and $l_{M}$, since the reasoning can be repeated for $\xi_{N}^{M \bowtie N}$ and $l_{N}$. 
In order to see that $\xi_{M}^{M \bowtie N}$ is automatically an action it suffices to follow these steps:

- show that $\eta_{M}^{M \bowtie N}=\left(q b 1_{M}\right) \circ \eta_{M}^{M+N}$ using the diagram

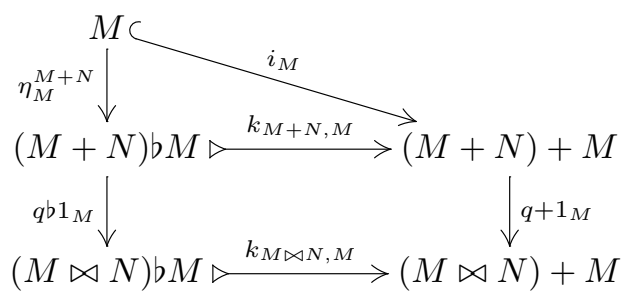

- show the first axiom:

$$
\xi_{M}^{M \bowtie N} \circ \eta_{M}^{M \bowtie N}=\xi_{M}^{M \bowtie N} \circ\left(q b 1_{M}\right) \circ \eta_{M}^{M+N}=\xi_{M}^{M+N} \circ \eta_{M}^{M+N}=1_{M} ;
$$

- $q b\left(q b 1_{M}\right)$ is a regular epimorphism due to Lemma 2.6;

- show the second axiom

$$
\xi_{M}^{M \bowtie N} \circ \mu_{M}^{M \bowtie N}=\xi_{M}^{M \bowtie N} \circ\left(1_{M \bowtie N} b \xi_{M}^{M \bowtie N}\right)
$$

using the commutativity of the outer rectangle

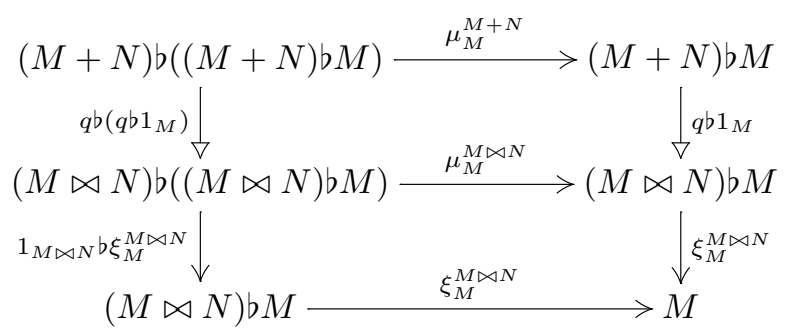

which follows from one of the axioms for the action $\xi_{M}^{M+N}$ (namely, the diagram on the right in Definition 2.15).

It remains to be shown that $\left(M \stackrel{l_{M}}{\longrightarrow} M \bowtie N, \xi_{M}^{M \bowtie N}\right)$ is indeed a crossed module. This amounts to the commutativity of the squares

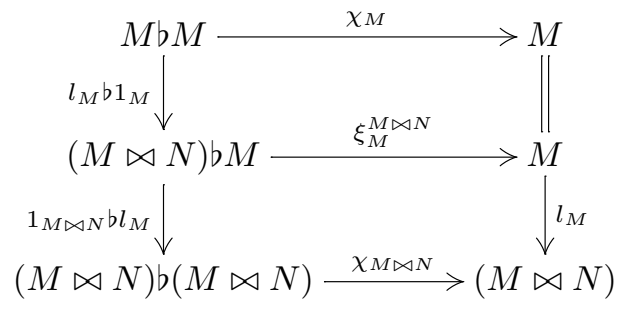

For the upper square we have the chain of equalities

$$
\xi_{M}^{M \bowtie N} \circ\left(l_{M} b 1_{M}\right)=\xi_{M}^{M \bowtie N} \circ\left(q b 1_{M}\right) \circ\left(i_{M} b 1_{M}\right)=\xi_{M}^{M+N} \circ\left(i_{M} b 1_{M}\right)=\chi_{M} .
$$


In order to show that the lower square commutes, consider the diagram

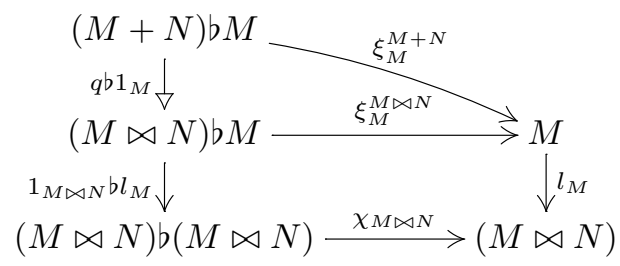

Since $q b 1_{M}$ is a regular epimorphism, it suffices to prove that the outer diagram is commutative. We decompose it as

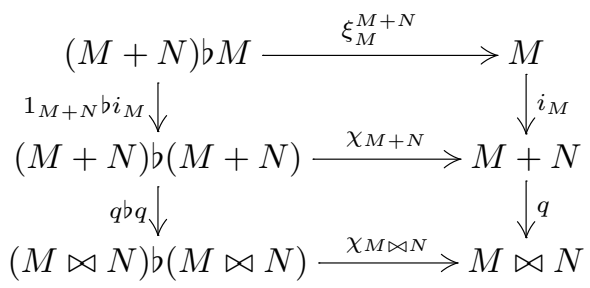

It is easy to check that the lower square commutes and thanks to this, by using Proposition 4.8, we find that the whole rectangle commutes.

Finally, we know that the actions $\xi_{N}^{M}$ and $\xi_{M}^{N}$ are in turn induced by $\xi_{M}^{M \bowtie N}$ and $\xi_{N}^{M \bowtie N}$ through the morphisms $l_{M}$ and $l_{N}$, that is

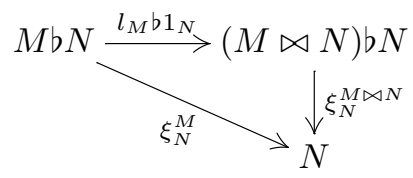

and

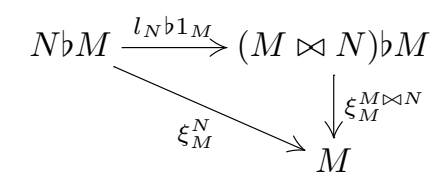

commute. This can be proved by using the definition of $l_{M}$ and $l_{N}$ and the commutativity of diagrams (CA.0), (19) and (20).

Remark 4.10. Notice that in the previous proposition we are implicitly using the (SH) condition: indeed we are using Definition 2.24, which requires (SH), as a definition for internal crossed modules.

Combining Proposition 4.3 and Proposition 4.9 we obtain the following characterisation of compatible actions, the main result of this article:

Theorem 4.11. In a semi-abelian category that satisfies $(\mathrm{SH})$, two actions $\xi_{N}^{M}$ and $\xi_{M}^{N}$ are compatible if and only if there exists an object $L$ endowed with crossed module structures

$$
\left(M \stackrel{\mu}{\rightarrow} L, \xi_{M}^{L}\right) \quad\left(N \stackrel{\nu}{\rightarrow} L, \xi_{N}^{L}\right)
$$

which, via the commutative triangles
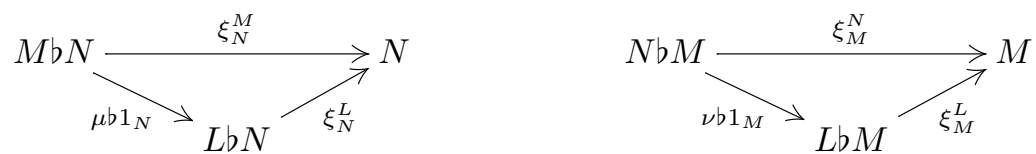

induce the given actions. 
As a consequence, our definition of compatible internal actions is indeed an extension of the particular definitions for groups and Lie algebras.

Corollary 4.12. In Grp Definition 4.1 coincides with Definition 3.3.

Proof. This is a combination of Theorem 4.11 with Proposition 3.10.

Corollary 4.13. The definition of compatible actions of Lie algebras given in [14] coincides with Definition 4.1 restricted to the category $\mathbf{L i e}_{R}$.

Proof. This is obtained through Theorem 4.11 by using Theorem 2.17 from [13].

\section{Universal properties of the Peiffer product}

The Peiffer product $M \bowtie N$ is the universal way to associate a coterminal pair of crossed modules to a pair of compatible actions.

Proposition 5.1. Consider a pair of compatible actions $\xi_{N}^{M}$ and $\xi_{M}^{N}$ and the pairs of coterminal crossed modules inducing them. The pair given by the Peiffer product is the universal one, in the sense that it is initial: for any pair of crossed modules

$$
\left(M \stackrel{\mu}{\rightarrow} L, \xi_{M}^{L}\right) \quad\left(N \stackrel{\nu}{\rightarrow} L, \xi_{N}^{L}\right)
$$

inducing $\xi_{N}^{M}$ and $\xi_{M}^{N}$ there exists a unique morphism $\left|{ }_{\nu}^{\mu}\right|: M \bowtie N \rightarrow L$ making the diagram

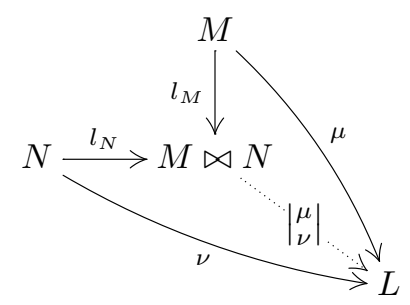

commute.

Proof. It suffices to show that $\left(\begin{array}{c}\mu \\ \nu\end{array}\right): M+N \rightarrow L$ coequalises the two morphisms defining $M \bowtie N$. Indeed that would give us a unique morphism $\left|{ }_{\nu}^{\mu}\right|$ such that

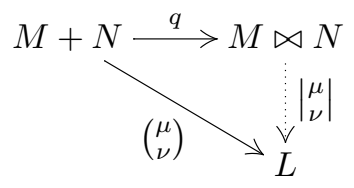

and then by precomposing with the inclusion we would get

$$
\mu=\left(\begin{array}{c}
\mu \\
\nu
\end{array}\right) \circ i_{M}=\left|\begin{array}{c}
\mu \\
\nu
\end{array}\right| \circ q \circ i_{M}=\left|\begin{array}{c}
\mu \\
\nu
\end{array}\right| \circ l_{M}, \quad \nu=\left(\begin{array}{c}
\mu \\
\nu
\end{array}\right) \circ i_{N}=\left|\begin{array}{c}
\mu \\
\nu
\end{array}\right| \circ q \circ i_{N}=\left|\begin{array}{c}
\mu \\
\nu
\end{array}\right| \circ l_{N} .
$$

Therefore we have to show that the two compositions

$$
(N b M)+(M b N) \frac{\left(\begin{array}{l}
k_{N, M} \\
k_{M, N}
\end{array}\right)}{\xi_{M}^{N}+\xi_{N}^{M}} \longrightarrow M+N \stackrel{\left(\begin{array}{c}
\mu \\
\nu
\end{array}\right)}{\longrightarrow} L L
$$


are equal. This is done via the chain of equalities

$$
\left.\left(\begin{array}{c}
\mu \\
\nu
\end{array}\right) \circ\left(\xi_{M}^{N}+\xi_{N}^{M}\right)=\left(\begin{array}{c}
\mu \circ \xi_{M}^{L} \circ \nu b 1_{M} \\
\nu \circ \xi_{N}^{L} \circ \mu b 1_{N}
\end{array}\right)=\left(\begin{array}{c}
\mu \\
\nu \\
\mu \\
\nu
\end{array}\right) \circ k_{N, M}\right)=\left(\begin{array}{c}
\mu \\
\nu
\end{array}\right) \circ\left(\begin{array}{c}
k_{N, M} \\
k_{M, N}
\end{array}\right)
$$

Lemma 5.2. Consider two pairs of coterminal crossed modules

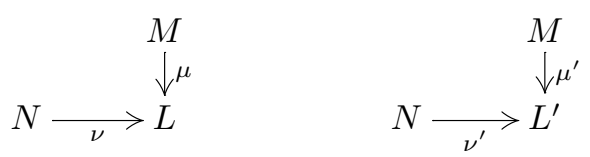

such that they induce the same actions between $M$ and $N$, that is such that the diagrams
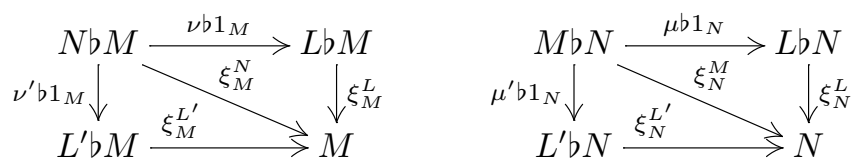

commute. Up to isomorphism, they induce the same Peiffer product $M \bowtie N$.

Proof. The induced actions $\xi_{M}^{M+N}$ and $\xi_{M}^{\prime M+N}$ (resp. $\xi_{N}^{M+N}$ and $\xi_{N}^{M+N}$ ) coincide when restricted to $M b M, N b M$ and $M \diamond N \diamond M(\operatorname{resp} . M b N, N b N$ and $M \diamond N \diamond N)$, therefore it suffices to use Remark 4.2 to obtain that $\xi_{M}^{M+N}=\xi_{M}^{\prime M+N}\left(\operatorname{resp} . \xi_{N}^{M+N}=\right.$ $\xi_{N}^{\prime M+N}$ ). As a consequence they induce isomorphic Peiffer products and isomorphic crossed module structures.

Finally, we use Proposition 4.5 to show the link between our definition of Peiffer product and the one given in [11].

Remark 5.3. We know from Proposition 3.2 in [11] that, as soon as $\left(M \stackrel{\mu}{\rightarrow} L, \xi_{M}^{L}\right)$ and $\left(N \stackrel{\nu}{\rightarrow} L, \xi_{N}^{L}\right)$ are (pre)crossed modules, we have induced actions of $L$ on $M \rtimes N$ and $N \rtimes M$ with corresponding (pre)crossed module structures. In general this is not true for $M \bowtie N$, but if $\mathbb{A}$ is algebraically coherent, by Proposition 4.1 and Proposition 4.3 in [11], and by Proposition 4.5 we obtain that our definition of Peiffer product coincides with the one given by Cigoli, Mantovani and Metere: consequently, $M \bowtie N$ is endowed with a precrossed module structure $\left(\left|{ }_{\nu}^{\mu}\right|: M \bowtie N \rightarrow L, \psi_{M \bowtie N}\right)$ as soon as $M$ and $N$ are so. Finally, when $\mathbb{A}$ satisfies the condition (UA) as well (see [11] for more on this condition), Theorem 5.2 in [11] tells us that the Peiffer product precrossed module turns out to be a crossed module as soon as $M$ and $N$ are so. Actually then it is the coproduct of $\left(M \stackrel{\mu}{\rightarrow} L, \xi_{M}^{L}\right)$ and $\left(N \stackrel{\nu}{\rightarrow} L, \xi_{N}^{L}\right)$ in the category $\operatorname{XMod}_{L}(\mathbb{A})$ of $L$-crossed modules in $\mathbb{A}$.

Remark 5.4. We do not know whether $L$ acts on $M \bowtie N$ when $\mathbb{A}$ is not algebraically coherent. And even if so, it is not clear to us whether this action defines a precrossed module structure.

\section{Acknowledgments}

We would like to thank the referee for detailed comments and suggestions which helped us to improve the text. 


\section{References}

[1] F. Borceux and D. Bourn, Mal'cev, protomodular, homological and semi-abelian categories, Mathematics and its Applications, vol. 566, Kluwer Academic Publishers, Dordrecht, 2004.

[2] F. Borceux, G. Janelidze, and G.M. Kelly, Internal object actions, Comment. Math. Univ. Carolin. 46 (2005), no. 2, 235-255.

[3] D. Bourn, $3 \times 3$ lemma and protomodularity, J. Algebra 236 (2001), no. 2, 778-795.

[4] D. Bourn, The denormalized $3 \times 3$ lemma, J. Pure Appl. Algebra 177 (2003), no. $2,113-129$.

[5] D. Bourn and G. Janelidze, Protomodularity, descent, and semidirect products, Theory Appl. Categ. 4 (1998), no. 2, 37-46.

[6] R. Brown and J.-L. Loday, Van Kampen theorems for diagrams of spaces, Topology 26 (1987), no. 3, 311-335.

[7] A. Carboni and G. Janelidze, Smash product of pointed objects in lextensive categories, J. Pure Appl. Algebra 183 (2003), 27-43.

[8] J.M. Casas, E. Khmaladze, and N. Pacheco Rego, A non-abelian tensor product of Hom-Lie algebras, Bull. Malays. Math. Sci. Soc. 40 (2017), no. 3, 1035-1054.

[9] J.M. Casas, E. Khmaladze, and N. Pacheco Rego, A non-abelian Hom-Leibniz tensor product and applications, Linear Multilinear Algebra 66 (2018), no. 6, 1133-1152.

[10] A.S. Cigoli, J.R.A. Gray, and T. Van der Linden, Algebraically coherent categories, Theory Appl. Categ. 30 (2015), no. 54, 1864-1905.

[11] A.S. Cigoli, S. Mantovani, and G. Metere, Peiffer product and Peiffer commutator for internal pre-crossed modules, Homology Homotopy Appl. 19 (2017), no. 1, 181-207.

[12] D. di Micco and T. Van der Linden, An intrinsic approach to the non-abelian tensor product via internal crossed squares, preprint arXiv:1911.08781, Nov 2019.

[13] D. di Micco, Compatible actions of Lie algebras, Comm. Algebra, 48, no. 2, $548-563$.

[14] G.J. Ellis, A nonabelian tensor product of Lie algebras, Glasgow Math. J. 33 (1991), no. 1, 101-120.

[15] M. Gerstenhaber, A categorical setting for the Baer extension theory, Appl. Categorical Algebra, Proc. Sympos. Pure Math. 17 (1970), 50-64.

[16] N.D. Gilbert and P.J. Higgins, The nonabelian tensor product of groups and related constructions, Glasgow Math. J. 31 (1989), no. 1, 17-29.

[17] A.V. Gnedbaye, A non-abelian tensor product of Leibniz algebras, Ann. Inst. Fourier (Grenoble) 49 (1999), no. 4, 1149-1177.

[18] M. Hartl and B. Loiseau, On actions and strict actions in homological categories, Theory Appl. Categ. 27 (2013), no. 15, 347-392.

[19] M. Hartl and T. Van der Linden, The ternary commutator obstruction for internal crossed modules, Adv. Math. 232 (2013), 571-607. 
[20] P.J. Higgins, Groups with multiple operators, Proc. Lond. Math. Soc. (3) 6 (1956), no. 3, 366-416.

[21] S.A. Huq, Commutator, nilpotency and solvability in categories, Q. J. Math. 19 (1968), no. 2, 363-389.

[22] N. Inassaridze, Nonabelian tensor products and nonabelian homology of groups, J. Pure Appl. Algebra 112 (1996), no. 2, 191-205.

[23] N. Inassaridze, Some aspects of homotopic algebra and non-abelian (co)homology theories, J. Math. Sci. (N. Y.) 213 (2016), no. 1-129.

[24] G. Janelidze, Internal crossed modules, Georgian Math. J. 10 (2003), no. 1, 99-114.

[25] G. Janelidze, L. Márki, and W. Tholen, Semi-abelian categories, J. Pure Appl. Algebra 168 (2002), no. 2-3, 367-386.

[26] E. Khmaladze, Non-abelian tensor and exterior products modulo $q$ and universal q-central relative extension of Lie algebras, Homology Homotopy Appl. 1 (1999), 187-204.

[27] S. Mantovani and G. Metere, Normalities and commutators, J. Algebra 324 (2010), no. 9, 2568-2588.

[28] N. Martins-Ferreira and T. Van der Linden, A note on the "Smith is Huq" condition, Appl. Categ. Structures 20 (2012), no. 2, 175-187.

[29] N. Martins-Ferreira and M. Sobral, On categories with semidirect products, J. Pure Appl. Algebra 216 (2012), no. 8-9, 1968-1975.

[30] G. Orzech, Obstruction theory in algebraic categories I and II, J. Pure Appl. Algebra 2 (1972), 287-314 and 315-340.

[31] M.C. Pedicchio, A categorical approach to commutator theory, J. Algebra 177 (1995), no. 3, 647-657.

[32] J.H.C. Whitehead, On adding relations to homotopy groups, Ann. of Math. (2) 42 (1941), no. 2, 409-428.

Davide di Micco davide.dimicco@unimi.it

Università degli Studi di Milano, Via Saldini 50, 20133 Milano, Italy

Tim Van der Linden tim.vanderlinden@uclouvain.be

Institut de Recherche en Mathématique et Physique, Université catholique de Louvain, chemin du cyclotron 2 bte L7.01.02, 1348 Louvain-la-Neuve, Belgium 